\title{
Microscopic Origin of Universality in Casimir Forces
}

\author{
Pascal R. Buenzli ${ }^{1,2}$ and Philippe A. Martin ${ }^{1}$
}

Received July 30, 2004; accepted September 28, 2004

\begin{abstract}
The microscopic mechanisms for universality of Casimir forces between macroscopic conductors are displayed in a model of classical charged fluids. The model consists of two slabs in empty space at distance $d$ containing classical charged particles in thermal equilibrium (plasma, electrolyte). A direct computation of the average force per unit surface yields, at large distance, the usual form of the Casimir force in the classical limit (up to a factor 2 due to the fact that the model does not incorporate the magnetic part of the force). Universality originates from perfect screening sum rules obeyed by the microscopic charge correlations in conductors. If one of the slabs is replaced by a macroscopic dielectric medium, the result of Lifshitz theory for the force is retrieved. The techniques used are Mayer expansions and integral equations for charged fluids.
\end{abstract}

KEY WORDS: Casimir forces; classical charged fluid; universality; Mayer expansion.

\section{INTRODUCTION}

It is well known that the fluctuation-induced forces between macroscopic conductors have a universal character: they only depend on the shapes of the bodies, but not on their material constitution. This observation originates from the celebrated paper of H. B. G. Casimir calculating the force between two parallel metallic plates due to the fluctuations of the quantum electromagnetic field in vacuum at zero temperature. The literature produced since then is so vast that we will only quote in the sequel a number of papers relevant to our purpose. Many references can be found for instances in the books and reviews. ${ }^{(1-4)}$

\footnotetext{
${ }^{1}$ Institute of Theoretical Physics, Swiss Federal Institute of Technology Lausanne, CH-1015, Lausanne EPFL, Switzerland.

${ }^{2}$ E-mail: pascal.buenzli@epfl.ch
} 
Regarding the extension of Casimir's result to non zero temperature $T$, Balian and Duplantier provide the general form of the free energy in presence of ideal conductors of arbitrary shapes. ${ }^{(5)}$ The theory of Lifshitz and coworkers generalizes the calculations to dielectric bodies characterized by their dielectric functions. ${ }^{(6,7)}$ (see also Ref. ${ }^{(9)}$ ). The ideal conductor situation can be recovered from the latter theory by letting the dielectric constants tend to infinity. From these studies one can obtain the asymptotic behaviour of the attractive force between two planar conductors at distance $d$ at high temperature (or equivalently at large separation $d)^{3}$

$$
f \sim \frac{k_{B} T \zeta(3)}{4 \pi d^{3}}, \quad d \rightarrow \infty
$$

where $\zeta$ is the Riemann $\zeta$-function. In this regime the force is exclusively due to thermal fluctuations and the result may be called classical since it does not depend on Planck's constant. In the above mentioned theories, the conductors are treated at the level of macroscopic physics. In fact they are represented by surfaces, called ideal conductors, on which the electromagnetic field has to satisfy the metallic boundary conditions. The purpose of this work is to gain an understanding of the microscopic mechanisms in the conductor that lead to the universality of the force (1).

To this end we analyse a simple model where the conductors are described in fully microscopic terms. The conductors consist of two slabs at distance $d$ containing fluids of classical charges (e.g. classical electrolytes or plasmas). The slabs are globally neutral but their material composition (charges and masses of the particles) can be different. The space external to the slabs is empty. The system of the two slabs, considered as a whole, is at thermal equilibrium with a Gibbs weight that includes pairwise interactions between all the particles, consisting of Coulomb potentials plus short-range repulsions. In this setting we present an exact computation of the asymptotic behaviour of the average force per unit surface between the two (infinitely thick) slabs giving

$$
\langle f\rangle \sim \frac{k_{B} T \zeta(3)}{8 \pi d^{3}}, \quad d \rightarrow \infty .
$$

It is also checked that (2) still holds for slabs of finite thickness (Appendix A).

One may notice that the usual approaches consider the fluctuating electromagnetic fields as primary objects. The thermal fluctuations of

\footnotetext{
${ }^{3}$ The relevant dimensionless parameter is $d k_{B} T / \hbar c, c$ is the speed of light, $\hbar$ the Planck constant, $k_{B}$ the Boltzmann constant.
} 
these fields originate from the fact that they are in equilibrium with the matter constituting the conductors, but as a consequence of universality, the microscopic degrees of freedom of the charges in the conductors do not need to be explicitly incorporated in the description. Here we adopt another point of view: we start from the thermal configurational fluctuations of the charges to provide a direct calculation of the average force without recourse to the field statistics. Then the origin of universality can be traced back to the specific sum rules obeyed by the correlations of Coulombic matter. ${ }^{(10)}$

Universal properties of a variety of classical models of conductor have been studied in Refs. 11 and 12. In Ref. 11, the authors consider a statistical mechanical system of charges confined to a plane at distance $d$ of another ideal planar conductor and establish the result (2). In Ref. 12, they show that replacing the above ideal conductor by fluctuating charges does not alter (2). A recent work $^{(13)}$ considers the situation where the space between the two slabs is filled by a third Coulomb fluid, causing a screening of the Casimir force.

The value (1) arising from the electromagnetic field fluctuations calculations is twice larger than that obtained in the purely electrostatic models considered here, as well as in Refs. 11-13. This point has been the subject of several discussions in the literature, in particular in Refs. 8 and 9. In Ref. 8, Schwinger performs a calculation of the Casimir force mediated by scalar photons (corresponding to the sole electric degree of freedom of an electromagnetic wave) leading to the result (2). In Ref. 9, the authors show that taking the magnetic degree of freedom of the field property into account multiplies the expression (2) by 2 . In its very formulation our model does not include the magnetic part of the Lorentz force induced by fluctuating currents in the conductors, whose effect has the same magnitude as that of the Coulomb force. Although such purely electrostatic models of conductors do not account for the physically correct value of the force at large distance, they already nicely reveal the microscopic mechanisms occurring in conductors that guarantee its universality.

The calculation of the force requires the knowledge of the charge correlation function across the two slabs separation, which is the main object of our study. At large separation it remarkably factorizes into three parts. There is a first factor independent of the slabs' material constitution and two other factors, each solely associated to one of the conductors. More precisely, the latter factors involve the charge density of the screening cloud induced by a charge located at the boundary of a single conductor in empty space. Then the universality of the force results from the perfect screening sum rule that holds in any conducting phase. 
In this work, we use the technique of Mayer expansion and integral equations for charged fluids. In Section 2, we specify the system under study and express the force by unit surface between two infinitely extended slabs in terms of the microscopic charge correlation between them, taking the existence of the thermodynamic limit for granted.

The general formalism used is recalled in Section 3: the charge correlation function is written in terms of the Ursell function subjected to a Mayer expansion. The prototype graphs entering in this expansion involve screened Coulomb bonds resulting from chain summations (Debye-Hückel mean-field potential) and density weights at vertices ${ }^{(14,15)}$. The weights are the exact inhomogeneous densities that have to be self-consistently determined from the first BGY equation. We do not treat here the full self-consistent problem because it turns out that the detailed structure of density profiles is not needed (see Refs. 16 and 17 for a thorough study of density profiles near boundaries). We only have to introduce weak and plausible assumptions on the convergence of the profiles to their bulk value.

It is shown in Section 4 that the asymptotic value (2) of the force is already obtained at the level of the Debye-Hückel theory. The main tool is the explicit form of the mean-field potential for piecewise-flat density profiles, related to the potential for structured profiles by an integral equation. The latter equation is shown to have a convergent perturbative solution in the weak-coupling regime. At large slab separation, the Debye-Hückel potential factorizes into potentials pertaining to individual plasmas obeying electroneutrality sum rules.

We establish in Section 5 that the theory beyond mean-field does not provide any additional contribution to the asymptotics (2). This is first done non-perturbatively with the help of integral equations corresponding to an appropriate dressing transformation of the Ursell function and under the mild assumption of integrable clustering. Finally the result is recovered once again by selecting and resumming the contribution of dominant Mayer graphs to the full charge correlation at large separation.

We also treat in Section 6 a variant situation where one of the slabs is replaced by an ideal macroscopic dielectric medium at distance $d$ (namely generating images of the plasma's fluctuating charges). Using the Green function of the Poisson equation with appropriate dielectric boundary conditions, the Lifshitz result for the mean force is retrieved and reduces to (2) as the dielectric constant tends to infinity. It is interesting to observe that it is sufficient for the fluctuations to occur only in one of the bodies to generate the same asymptotic behaviour. 
We will come back to the inclusion of magnetic forces and to quantum models in future works.

\section{DESCRIPTION OF THE MODEL}

We consider two plasmas $A$ and $B$ of classical point charges confined to two planar slabs $\Lambda_{A}(L, a)$ and $\Lambda_{B}(L, b)$ in three-dimensional space. The slabs have thickness $a$ and $b$, surface $L^{2}$, and are separated by a distance $d$ :

$$
\begin{gathered}
\Lambda_{A}(L, a):=\left\{\mathbf{r}=(x, \mathbf{y}) \mid x \in[-a, 0], \quad \mathbf{y} \in\left[-\frac{L}{2}, \frac{L}{2}\right]^{2}\right\} \\
\Lambda_{B}(L, b):=\left\{\mathbf{r}=(x, \mathbf{y}) \mid x \in[d, d+b], \quad \mathbf{y} \in\left[-\frac{L}{2}, \frac{L}{2}\right]^{2}\right\} .
\end{gathered}
$$

The plasma $A(B)$ is made of charges $e_{\alpha}\left(e_{\beta}\right)$ of species $\alpha \in S_{A}\left(\beta \in S_{B}\right)$ where $S_{A}$ and $S_{B}$ are index sets for the species in $\Lambda_{A}(L, a)$ and $\Lambda_{B}(L, b)$ respectively. We assume both plasmas to be globally neutral, i.e. carrying no net charge,

$$
\sum_{a} e_{\alpha_{a}}=\sum_{b} e_{\beta_{b}}=0
$$

where $\sum_{a}\left(\sum_{b}\right)$ extends on all particles in $\Lambda_{A}(L, a)\left(\Lambda_{B}(L, b)\right)$. For a particle located at $\mathbf{r}$ we will use the generic notation $(\gamma \mathbf{r})$ where $\gamma \in S_{A}$ if $\mathbf{r} \in$ $\Lambda_{A}(L, a)$ and $\gamma \in S_{B}$ if $\mathbf{r} \in \Lambda_{B}(L, b)$. The space external to the slabs is supposed to have no electrical properties, its dielectric constant being taken equal to that of vacuum. The charges are confined in the slabs by hard walls that merely limit the available configuration space to the regions (3).

All particles interact via the two-body potential

$$
V\left(\gamma, \gamma^{\prime},\left|\mathbf{r}-\mathbf{r}^{\prime}\right|\right)=e_{\gamma} e_{\gamma^{\prime}} v\left(\mathbf{r}-\mathbf{r}^{\prime}\right)+v_{\mathrm{SR}}\left(\gamma, \gamma^{\prime},\left|\mathbf{r}-\mathbf{r}^{\prime}\right|\right),
$$

where $v\left(\mathbf{r}-\mathbf{r}^{\prime}\right)=1 /\left|\mathbf{r}-\mathbf{r}^{\prime}\right|$ is the Coulomb potential (in Gaussian units) and $v_{\mathrm{SR}}\left(\gamma, \gamma^{\prime}, \mathbf{r}-\mathbf{r}^{\prime}\right)$ is a short-range repulsive potential to prevent the collapse of opposite charges and guarantee the thermodynamic stability of the system.

The total potential energy $U$ consists in the sum of all pairwise interactions, separated into three contributions according to whether they take place between two particles of $A$, of $B$, or between a particle of $A$ and a particle of $B$ :

$$
U=U_{A}+U_{B}+U_{A B} .
$$


On the microscopic level, the force between configurations of charges in the two plasmas is the sum of all pairwise forces exerted by the particles of $B$ on the particles of $A$ :

$$
\begin{gathered}
\mathbf{F}_{\Lambda_{B} \rightarrow \Lambda_{A}}:=\sum_{a} \sum_{b}\left[e_{\alpha_{a}} e_{\beta_{b}} \frac{\mathbf{r}_{a}-\mathbf{r}_{b}}{\left|\mathbf{r}_{a}-\mathbf{r}_{b}\right|^{3}}+\mathbf{F}_{\mathrm{SR}}\left(\alpha_{a}, \beta_{b}, \mathbf{r}_{a}-\mathbf{r}_{b}\right)\right] \\
\mathbf{r}_{a} \in \Lambda_{A}(L, a), \quad \mathbf{r}_{b} \in \Lambda_{B}(L, b)
\end{gathered}
$$

and $\mathbf{F}_{\mathrm{SR}}$ is the force associated to the short-range potential $v_{\mathrm{SR}}$. For simplicity we assume that the range of $v_{\mathrm{SR}}$ is finite so that $\mathbf{F}_{\mathrm{SR}}\left(\alpha_{a}, \beta_{b}, \mathbf{r}_{a}-\mathbf{r}_{b}\right)$ vanishes as soon as $d$ is large enough, and we will omit it in the following.

Both plasmas are supposed to be in thermal equilibrium at the same temperature $T$. The statistical average $\langle\cdots\rangle_{L}$ is defined in terms of the Gibbs weight $\exp (-\beta U), \beta=\left(k_{B} T\right)^{-1}$, associated with the total energy (6). There is no need to explicitly specify the ensemble used here (canonical or grand canonical) provided that the global neutrality constraint (4) is taken into account. The average particle densities $\rho_{L}(\gamma \mathbf{r})$ are expressed as averages of the microscopic particle densities $\hat{\rho}(\gamma \mathbf{r})=\sum_{i} \delta_{\gamma \gamma_{i}} \delta\left(\mathbf{r}-\mathbf{r}_{i}\right)$ where the sum runs over all particles

$$
\rho_{L}(\gamma \mathbf{r})=\langle\hat{\rho}(\gamma \mathbf{r})\rangle_{L}
$$

We keep the index $L$ to remember that averages are taken for the finite-volume slabs (3). Hence expressing the sums in (7) as integrals on particle densities $\hat{\rho}(\gamma \mathbf{r})$, the average force reads

$$
\langle\mathbf{F}\rangle_{L}=\int_{\Lambda_{A}(L)} d \mathbf{r} \int_{\Lambda_{B}(L)} d \mathbf{r}^{\prime} \frac{\mathbf{r}-\mathbf{r}^{\prime}}{\left|\mathbf{r}-\mathbf{r}^{\prime}\right|^{3}} c_{L}\left(\mathbf{r}, \mathbf{r}^{\prime}\right)
$$

where $c_{L}\left(\mathbf{r}, \mathbf{r}^{\prime}\right)$ is the two-point charge correlation function

$$
c_{L}\left(\mathbf{r}, \mathbf{r}^{\prime}\right)=\left\langle\hat{c}(\mathbf{r}) \hat{c}\left(\mathbf{r}^{\prime}\right)\right\rangle_{L}, \quad \hat{c}(\mathbf{r})=\sum_{\gamma} e_{\gamma} \hat{\rho}(\gamma \mathbf{r}) .
$$

We now consider the average force by unit surface between two infinitely extended slabs at distance $d$ by letting their transverse dimension $L$ tend to infinity. We assume that the plasma phases are in fluid states homogeneous and isotropic in the $\mathbf{y}$ directions, namely the charge correlation has an infinite-volume limit of the form

$$
\lim _{L \rightarrow \infty} c_{L}\left(\mathbf{r}, \mathbf{r}^{\prime}\right)=\left\langle\hat{c}(\mathbf{r}) \hat{c}\left(\mathbf{r}^{\prime}\right)\right\rangle=c\left(x, x^{\prime},\left|\mathbf{y}-\mathbf{y}^{\prime}\right|\right) .
$$


For symmetry reasons, $\langle\mathbf{F}\rangle_{L}$ has no transverse component and is directed along the $x$ axis perpendicular to the plates. We therefore consider the $x$-component of the force per unit surface

$$
\begin{aligned}
\langle f\rangle & :=\lim _{L \rightarrow \infty} \frac{\left\langle F_{x}\right\rangle_{L}}{L^{2}} \\
& =\lim _{L \rightarrow \infty} \frac{1}{L^{2}} \int_{L^{2}} d \mathbf{y}\left(\int_{-a}^{0} d x \int_{d}^{d+b} d x^{\prime} \int_{L^{2}} d \mathbf{y}^{\prime} \frac{x-x^{\prime}}{\left|\mathbf{r}-\mathbf{r}^{\prime}\right|^{3}} c_{L}\left(x, \mathbf{y}, x^{\prime}, \mathbf{y}^{\prime}\right)\right) \\
& =\int_{-a}^{0} d x \int_{d}^{d+b} d x^{\prime} \int d \mathbf{y} \frac{x-x^{\prime}}{\left[\left(x-x^{\prime}\right)^{2}+|\mathbf{y}|^{2}\right]^{3 / 2}} c\left(x, x^{\prime},|\mathbf{y}|\right) .
\end{aligned}
$$

The last line results from the $\mathbf{y}$ translational invariance of the integrand in the limit $L \rightarrow \infty$. We do not justify the existence of the limit here (which depends on a uniform control of $c_{L}\left(x, \mathbf{y}, x^{\prime}, \mathbf{y}^{\prime}\right)$ as $\left.\left|\mathbf{y}-\mathbf{y}^{\prime}\right| \rightarrow \infty\right)$, but it will be clear from the subsequent calculations that (12) is a well defined quantity, at least in the weak-coupling regime.

Formula (12) remains valid if one replaces $c\left(x, x^{\prime},|\mathbf{y}|\right)$ by the truncated charge-charge correlation function

$$
S\left(x, x^{\prime}, \mathbf{y}\right)=\left\langle\hat{c}(\mathbf{r}) \hat{c}\left(\mathbf{r}^{\prime}\right)\right\rangle-\langle\hat{c}(\mathbf{r})\rangle\left\langle\hat{c}\left(\mathbf{r}^{\prime}\right)\right\rangle, \quad \mathbf{r}=(x, \mathbf{y}), \quad \mathbf{r}^{\prime}=\left(x^{\prime}, \mathbf{0}\right)
$$

with $\hat{c}(\mathbf{r})$ the microscopic charge density as in (10). Indeed, the $\mathbf{y}$-Fourier transform of the Coulomb force reads

$$
\int d \mathbf{y} \mathrm{e}^{-i \mathbf{k} \cdot \mathbf{y}} \frac{x-x^{\prime}}{\left[\left(x-x^{\prime}\right)^{2}+|\mathbf{y}|^{2}\right]^{3 / 2}}=2 \pi \operatorname{sign}\left(x-x^{\prime}\right) \mathrm{e}^{-k\left|x-x^{\prime}\right|}
$$

and reduces to $-2 \pi$ when $\mathbf{k}=\mathbf{0}$ and $x<x^{\prime}$. This implies that the charge density profile $\langle\hat{c}(\mathbf{r})\rangle=c(x)$ does not contribute to the force because of the global neutrality of both plasmas

$$
\int_{-a}^{0} d x c(x)=\int_{d}^{d+b} d x c(x)=0
$$

To take full advantage of the translational invariance in the $\mathbf{y}$ direction we represent the $\mathbf{y}$-integral in (12) in Fourier space:

$$
\langle f\rangle=-\frac{1}{2 \pi} \int_{-a}^{0} d x \int_{d}^{d+b} d x^{\prime} \int d \mathbf{k} \mathrm{e}^{-k\left|x-x^{\prime}\right|} S\left(x, x^{\prime}, \mathbf{k}\right),
$$


where $k=|\mathbf{k}|$ and $S\left(x, x^{\prime}, \mathbf{k}\right)=\int d \mathbf{y} e^{-i \mathbf{k} \cdot \mathbf{y}} S\left(x, x^{\prime}, \mathbf{y}\right)$. The dependence of $\langle f\rangle=\langle f\rangle(d)$ on the separation $d$ between the two slabs occurs in the integration limits in (16) as well as in the charge correlation function $S\left(x, x^{\prime}, \mathbf{k}\right)$. The $d$ dependence of the correlations between the two slabs $A$ and $B$ originates itself from the Coulomb interaction term $U_{A B}$ occurring in the total Gibbs thermal weight. The object of the next sections is to determine the asymptotic behaviour of $\langle f\rangle(d)$ as $d \rightarrow \infty$.

\section{MAYER SERIES FOR INHOMOGENEOUS CHARGED FLUIDS}

We briefly summarise the methods that we use to calculate the charge-charge correlation function of our system. Let us consider a general charged fluid in presence of spatial inhomogeneities caused by an external potential $\Psi^{\mathrm{ext}}(\gamma \mathbf{r})$, e.g. wall potentials confining the system in some region of space. Hard walls without electrical properties (infinite potentials) can be implemented by simply declaring that the density vanishes in the forbidden regions.

It is well-known (e.g. Ref. 18) that the two-point Ursell function, related to the densities $\rho(i), \rho(j)$ and the two-particle density $\rho(i, j)$

$$
h(i, j):=\frac{\rho(i, j)}{\rho(i) \rho(j)}-1,
$$

can be expanded in a formal power series of the densities by means of Mayer graphs. The basic Mayer bonds are

$$
f(i, j)=e^{-\beta V(i, j)}-1
$$

where $V(i, j)$ is the potential (5) and the weights at vertices are the densities $\rho(i)$. Here $i$ is a shorthand notation for the point $\left(\gamma_{i} \mathbf{r}_{i}\right)$ in configuration space, and integration on configurations $\sum_{\gamma_{i}} \int d \mathbf{r}_{i}$ includes the summation on particle species. Diagrams have two root points $i$ and $j$ and $m$ internal points which have to be integrated over. Each pair of points is linked by at most one $f$-bond and there are no articulation points. ${ }^{4}$ Because of the long-range of Coulomb interaction, the integrals occurring in every diagram diverge in the thermodynamic limit. It is therefore necessary to introduce the screened mean-field potential $\Phi\left(\mathbf{r}_{i}, \mathbf{r}_{j}\right)$ as usual by resumming the chains built with pure Coulombic interaction bonds

\footnotetext{
${ }^{4} \mathrm{An}$ articulation point, when removed, splits the diagram into two pieces, at least one of which is disconnected from the root points.
} 
$-\beta e_{\gamma_{i}} e_{\gamma_{j}} v\left(\mathbf{r}_{i}-\mathbf{r}_{j}\right)$. Then replacing the bare Coulomb potential by the screened potential leads to a reorganisation of the diagrammatic expansion of the Ursell function resulting in the formula ${ }^{(15)}$

$$
h\left(\gamma \mathbf{r}, \gamma^{\prime} \mathbf{r}^{\prime}\right)=\sum_{\Pi} \frac{1}{S_{\Pi}} \sum_{\gamma_{1}, \ldots, \gamma_{m}} \int d \mathbf{r}_{1} \cdots d \mathbf{r}_{m} \rho\left(\gamma_{1} \mathbf{r}_{1}\right) \cdots \rho\left(\gamma_{m} \mathbf{r}_{m}\right) \prod_{\{i, j\} \in \Pi} \mathcal{F}(i, j)
$$

The first sum runs over all unlabelled topologically different connected diagrams $\Pi$ (called prototype graphs) with two root points $(\gamma \mathbf{r})$ and $\left(\gamma^{\prime} \mathbf{r}^{\prime}\right)$ and $m$ integrated internal points with density weights $(m$ ranges from 0 to $\infty) ; S_{\Pi}$ denotes the symmetry number of a diagram $\Pi$. Each pair of points is linked by at most one bond $\mathcal{F} \in\left\{F, F^{\mathrm{R}}\right\}$ and there are no articulation points. Moreover, convolutions of $F$ bonds are forbidden to avoid multiple counting of original Mayer graphs. The two possible bonds read

$$
\begin{aligned}
F(i, j)= & -\beta e_{\gamma_{i}} e_{\gamma_{j}} \Phi\left(\mathbf{r}_{i}, \mathbf{r}_{j}\right) \\
F^{\mathrm{R}}(i, j)= & \exp \left[-\beta e_{\gamma_{i}} e_{\gamma_{j}} \Phi\left(\mathbf{r}_{i}, \mathbf{r}_{j}\right)-\beta v_{\mathrm{SR}}\left(\gamma_{i}, \gamma_{j},\left|\mathbf{r}_{i}-\mathbf{r}_{j}\right|\right)\right] \\
& -1+\beta e_{\gamma_{i}} e_{\gamma_{j}} \Phi\left(\mathbf{r}_{i}, \mathbf{r}_{j}\right)
\end{aligned}
$$

These bonds are obtained in terms of the Debye-Hückel screened potential $\Phi$, which is symmetric and defined as the solution of the integral equation

$$
\Phi\left(\mathbf{r}, \mathbf{r}^{\prime}\right)=v\left(\mathbf{r}-\mathbf{r}^{\prime}\right)-\frac{1}{4 \pi} \int d \mathbf{r}_{1} \kappa^{2}\left(\mathbf{r}_{1}\right) v\left(\mathbf{r}-\mathbf{r}_{1}\right) \Phi\left(\mathbf{r}_{1}, \mathbf{r}^{\prime}\right)=\Phi\left(\mathbf{r}^{\prime}, \mathbf{r}\right)
$$

or equivalently of the differential equation

$$
\Delta \Phi\left(\mathbf{r}, \mathbf{r}^{\prime}\right)-\kappa^{2}(\mathbf{r}) \Phi\left(\mathbf{r}, \mathbf{r}^{\prime}\right)=-4 \pi \delta\left(\mathbf{r}-\mathbf{r}^{\prime}\right)
$$

supplemented by suitable boundary conditions. In (22) and (23)

$$
\kappa(\mathbf{r}):=\left(4 \pi \beta \sum_{\gamma} e_{\gamma}^{2} \rho(\gamma \mathbf{r})\right)^{1 / 2}
$$

can be interpreted as the local inverse Debye screening length in the inhomogeneous system. The bond $F^{\mathrm{R}}(i, j)$ includes the short-range contribution and the nonlinear Coulombic part of the original Mayer bond.

The densities $\rho(\gamma \mathbf{r})$ entering in (19) and (24) have to be determined self-consistently from the first equation of the Born-Green-Yvon hierarchy 
which links the one-point and the two-point functions. For charged systems, it takes the form ${ }^{(10)}$

$$
\begin{aligned}
\nabla \rho(\gamma \mathbf{r})= & -\beta e_{\gamma} \rho(\gamma \mathbf{r})\left[\nabla \Psi(\gamma \mathbf{r})+\int d \mathbf{r}^{\prime}\left(\sum_{\gamma^{\prime}} e_{\gamma^{\prime}} \rho\left(\gamma^{\prime} \mathbf{r}^{\prime}\right) h\left(\gamma \mathbf{r}, \gamma^{\prime} \mathbf{r}^{\prime}\right)\right) \nabla v\left(\mathbf{r}-\mathbf{r}^{\prime}\right)\right] \\
& -\beta \sum_{\gamma^{\prime}} \int d \mathbf{r}^{\prime} \rho\left(\gamma \mathbf{r}, \gamma^{\prime} \mathbf{r}^{\prime}\right) \nabla v_{\mathrm{SR}}\left(\gamma, \gamma^{\prime},\left|\mathbf{r}-\mathbf{r}^{\prime}\right|\right)
\end{aligned}
$$

where

$$
\Psi(\gamma \mathbf{r})=\Psi^{\mathrm{ext}}(\gamma \mathbf{r})+\int d \mathbf{r}^{\prime} c\left(\mathbf{r}^{\prime}\right) v\left(\mathbf{r}-\mathbf{r}^{\prime}\right)
$$

is the sum of the external potential and the electrostatic potential caused by the inhomogeneous mean charge density $c\left(\mathbf{r}^{\prime}\right)$ in the system. Hence the Ursell function (considered as a functional of the densities through its Mayer expansion (19)) together with (25) form a closed set of equations whose solution determines in principle the exact densities and two-particle correlations. The differential equation (25) has still to be supplemented with appropriate boundary conditions. For instance if the system is asymptotically uniform in some directions, one can fix the corresponding asymptotic bulk densities.

Finally, the charge-charge correlation function (13) is related to the Ursell function by

$$
S\left(\mathbf{r}, \mathbf{r}^{\prime}\right)=\sum_{\gamma, \gamma^{\prime}} e_{\gamma} e_{\gamma^{\prime}} \rho(\gamma \mathbf{r}) \rho\left(\gamma^{\prime} \mathbf{r}^{\prime}\right) h\left(\gamma \mathbf{r}, \gamma^{\prime} \mathbf{r}^{\prime}\right)+\delta\left(\mathbf{r}-\mathbf{r}^{\prime}\right) \sum_{\gamma} e_{\gamma}^{2} \rho(\gamma \mathbf{r})
$$

The second term in the r.h.s. of (27) is the contribution of coincident points.

We shall use the above formalism to calculate (27) as a function of the distance $d$ for two infinitely thick plasmas (i.e., $a \rightarrow \infty$ and $b \rightarrow \infty$ in (16)) and with hard walls at $x=0$ and $x=d$. In this situation we take $\Psi^{\mathrm{ext}}(\gamma \mathbf{r})=0$ for $x<0$ and $x>d$, and impose

$$
\begin{aligned}
\rho(\gamma x) & =0, \quad 0 \leqslant x \leqslant d, \\
\lim _{x \rightarrow-\infty} \rho(\gamma x) & =\rho_{A \gamma}, \quad \lim _{x \rightarrow \infty} \rho(\gamma x)=\rho_{B \gamma}
\end{aligned}
$$

where $\rho_{A \gamma}$ and $\rho_{B \gamma}$ are the bulk particle densities of plasmas $A$ and $B$. The contribution of coincident points does not enter into the force (16) 
since $\mathbf{r}$ and $\mathbf{r}^{\prime}$ are always at least separated by the distance $d$. Therefore, (16) reads

$$
\langle f\rangle=-\int_{-\infty}^{0} d x \int_{d}^{\infty} d x^{\prime} \int_{0}^{\infty} d k k e^{-k\left|x-x^{\prime}\right|} \sum_{\gamma, \gamma^{\prime}} e_{\gamma} e_{\gamma^{\prime}} \rho(\gamma x) \rho\left(\gamma^{\prime} x^{\prime}\right) h\left(\gamma x, \gamma^{\prime} x^{\prime}, \mathbf{k}\right)
$$

with $h\left(\gamma x, \gamma^{\prime} x^{\prime}, \mathbf{k}\right)$ the $\mathbf{y}$-Fourier transform of the Ursell function.

\section{DEBYE-HÜCKEL THEORY}

In this section, we show that the simplest contribution to $h\left(\gamma \mathbf{r}, \gamma^{\prime} \mathbf{r}^{\prime}\right)$ given by the sole bond $F$, namely,

$$
h^{\mathrm{DH}}\left(\gamma \mathbf{r}, \gamma^{\prime} \mathbf{r}^{\prime}\right)=-\beta e_{\gamma} e_{\gamma^{\prime}} \Phi\left(\mathbf{r}, \mathbf{r}^{\prime}\right)
$$

already leads to the asymptotic value (2) of the force. For this we have to find the screened potential by solving (23) (written in Fourier form) with the boundary conditions imposed by the slab geometries

$$
\left[\frac{\partial^{2}}{\partial x^{2}}-k^{2}-\kappa^{2}(x)\right] \Phi\left(x, x^{\prime}, \mathbf{k}\right)=-4 \pi \delta\left(x-x^{\prime}\right), \quad \kappa(x)=0,0<x<d,
$$

with $\Phi\left(x, x^{\prime}, \mathbf{k}\right)=\int d \mathbf{y} \mathrm{e}^{-i \mathbf{k} \cdot \mathbf{y}} \Phi\left(x, x^{\prime}, \mathbf{y}\right)$. The boundary conditions are $\Phi\left(x, x^{\prime}, \mathbf{k}\right)$ and $\partial \Phi\left(x, x^{\prime}, \mathbf{k}\right) / \partial x$ continuous at $x=0$ and $x=d$, and $\lim _{x \rightarrow \pm \infty} \Phi\left(x, x^{\prime}, \mathbf{k}\right)=0$. The density profiles entering in $\kappa^{2}(x)$ by (24) are not known (since they have to be determined by self-consistency from (25)), but we will not need their explicit form in the sequel. ${ }^{5}$ We only need to assume that their difference to bulk value is integrable:

$$
\begin{array}{ll}
\rho(\gamma x)-\rho_{A \gamma}=\mathcal{O}\left(\frac{1}{|x|^{1+\epsilon}}\right), \quad x \rightarrow-\infty, \\
\rho(\gamma x)-\rho_{B \gamma}=\mathcal{O}\left(\frac{1}{|x|^{1+\epsilon}}\right), \quad x \rightarrow \infty, \epsilon>0 .
\end{array}
$$

\footnotetext{
${ }^{5} \mathrm{~A}$ mean-field approximation to the densities could be obtained by replacing $h$ in (25) by $h^{\mathrm{DH}}$. We are not doing so here but deal throughout with the exact densities.
} 
Integrating (31) on $x$ leads to

$$
\int_{-\infty}^{\infty} d x \frac{\kappa^{2}(x)}{4 \pi} \Phi\left(x, x^{\prime}, \mathbf{k}\right)=1-\frac{k^{2}}{4 \pi} \int d x \Phi\left(x, x^{\prime}, \mathbf{k}\right) .
$$

In particular, for $\mathbf{k}=0$

$$
\int_{-\infty}^{\infty} d x \frac{\kappa^{2}(x)}{4 \pi} \Phi\left(x, x^{\prime}, \mathbf{k}=0\right)=1
$$

which is nothing else than the electroneutrality sum rule for the chargecharge correlation (13), (27) within the Debye regime $(30)^{(10)}$ :

$$
\int_{-\infty}^{\infty} d x \int d \mathbf{y} S^{D H}\left(x, x^{\prime}, \mathbf{y}\right)=0
$$

To solve (31) we first consider the simpler problem with piecewise-flat densities $\rho_{A \gamma}$ and $\rho_{B \gamma}$ in each plasma

$$
\begin{aligned}
& {\left[\frac{\partial^{2}}{\partial x^{2}}-k^{2}-\bar{\kappa}^{2}(x)\right] \varphi\left(x, x^{\prime}, \mathbf{k}\right)=-4 \pi \delta\left(x-x^{\prime}\right)} \\
& \bar{\kappa}(x)=\kappa_{A}, x<0, \quad \bar{\kappa}(x)=0,0<x<d, \quad \bar{\kappa}(x)=\kappa_{B}, x>d
\end{aligned}
$$

where

$$
\kappa_{A}=\left(4 \pi \beta \sum_{\alpha \in S_{A}} e_{\alpha}^{2} \rho_{A \alpha}\right)^{1 / 2}, \quad \kappa_{B}=\left(4 \pi \beta \sum_{\beta \in S_{B}} e_{\beta}^{2} \rho_{B} \beta\right)^{1 / 2}
$$

are the bulk inverse screening lengths. The boundary conditions are the same as for (31). Denoting by $\mathcal{L}$ the linear operator acting on $\Phi$ on the 1.h.s. of (31) and by $\overline{\mathcal{L}}$ the one acting similarly on $\varphi$, one has $\mathcal{L} \Phi(x)-$ $\overline{\mathcal{L}} \varphi(x)=\overline{\mathcal{L}}(\Phi-\varphi)(x)-u(x) \Phi(x)=0$, where $u(x)=\kappa^{2}(x)-\bar{\kappa}^{2}(x)$ represents the deviation of the density profiles to their bulk limiting values. Since $-\varphi / 4 \pi$ is the Green function of $\overline{\mathcal{L}}$, it follows that $\Phi\left(x, x^{\prime}, \mathbf{k}\right)$ and $\varphi\left(x, x^{\prime}, \mathbf{k}\right)$ are related by the integral equation

$$
\Phi\left(x, x^{\prime}, \mathbf{k}\right)=\varphi\left(x, x^{\prime}, \mathbf{k}\right)-\frac{1}{4 \pi} \int d s u(s) \varphi(x, s, \mathbf{k}) \Phi\left(s, x^{\prime}, \mathbf{k}\right)
$$


which expresses $\Phi\left(x, x^{\prime}, \mathbf{k}\right)$ as a perturbation of $\varphi\left(x, x^{\prime}, \mathbf{k}\right)$ by the inhomogeneity $u(x)$ of the plasmas' density profiles.

Solving (36) piecewise and connecting the solutions together yields ${ }^{6}$

$$
\varphi\left(x, x^{\prime}, \mathbf{k}\right)= \begin{cases}\varphi_{A A}\left(x, x^{\prime}, \mathbf{k}\right), & x, x^{\prime}<0, \\ \varphi_{A B}\left(x, x^{\prime}-d, \mathbf{k}\right), & x<0<d<x^{\prime}, \\ \varphi_{B B}\left(x-d, x^{\prime}-d, \mathbf{k}\right), & d<x, x^{\prime}\end{cases}
$$

with

$$
\begin{aligned}
\varphi_{A A}\left(x, x^{\prime}, \mathbf{k}\right) & =2 \pi \frac{\mathrm{e}^{-k_{A}\left|x-x^{\prime}\right|}}{k_{A}}+2 \pi \frac{\mathrm{e}^{-k_{A}\left|x+x^{\prime}\right|}}{k_{A}} \frac{\left(k_{A}-k\right)\left(k_{B}+k\right) \mathrm{e}^{k d}-\left(k_{A}+k\right)\left(k_{B}-k\right) \mathrm{e}^{-k d}}{\left(k_{A}+k\right)\left(k_{B}+k\right) \mathrm{e}^{k d}-\left(k_{A}-k\right)\left(k_{B}-k\right) \mathrm{e}^{-k d}}, \\
\varphi_{A B}\left(x, x^{\prime}, \mathbf{k}\right) & =\frac{8 \pi k \mathrm{e}^{-k_{A}|x|} \mathrm{e}^{-k_{B}\left|x^{\prime}\right|}}{\left(k_{A}+k\right)\left(k_{B}+k\right) \mathrm{e}^{k d}-\left(k_{A}-k\right)\left(k_{B}-k\right) \mathrm{e}^{-k d}}, \\
k_{A} & =\sqrt{k^{2}+\kappa_{A}^{2}}, \quad k_{B}=\sqrt{k^{2}+\kappa_{B}^{2}}
\end{aligned}
$$

The function $\varphi_{B B}\left(x, x^{\prime}, \mathbf{k}\right)$ is obtained by interchanging the indices $A$ and $B$ in (40). Notice that $\varphi\left(x, x^{\prime}, \mathbf{k}\right)=\varphi\left(x^{\prime}, x, \mathbf{k}\right)$ and is invariant under the symmetry $x \leftrightarrow d-x, x^{\prime} \leftrightarrow d-x^{\prime}, A \leftrightarrow B$.

We discuss a few properties of this solution. The first term in the r.h.s of (40) corresponds to the bulk Debye-Hückel potential whereas the second term is the modification due to the finite boundaries of both plasmas $A$ and $B$. As $d \rightarrow \infty, \varphi_{A A}\left(x, x^{\prime}, \mathbf{k}\right)$ reduces to the well-known DebyeHückel potential $\varphi_{A}^{0}\left(x, x^{\prime}, \mathbf{k}\right)$ of a single semi-infinite plasma in the region $x<0$ (see formula (24) in Ref. 19 and Ref. 20)

$$
\lim _{d \rightarrow \infty} \varphi_{A A}\left(x, x^{\prime}, \mathbf{k}\right)=2 \pi \frac{\mathrm{e}^{-k_{A}\left|x-x^{\prime}\right|}}{k_{A}}+2 \pi \frac{k_{A}-k}{k_{A}+k} \frac{\mathrm{e}^{-k_{A}\left|x+x^{\prime}\right|}}{k_{A}}=\varphi_{A}^{0}\left(x, x^{\prime}, \mathbf{k}\right)
$$

uniformly with respect to $\mathbf{k}$. One observes that $\varphi\left(x, x^{\prime}, \mathbf{k}\right)$ is an even, infinitely differentiable function of $|\mathbf{k}|$, implying that $\varphi\left(x, x^{\prime}, \mathbf{y}\right)$ decays along walls directions faster than any inverse power of $y$. This is to be contrasted with the small $\mathbf{k}$ behaviour of the function (43) which has a non analytic $|\mathbf{k}|$ term leading to the algebraic decay $y^{-3}$ along the wall, ${ }^{(20)}$; see also Ref. 10, Section III.C.2).

\footnotetext{
${ }^{6}$ The functions $\varphi_{A B}, \varphi_{B B}$ (depending on $d$ ) refer to the system of the two plasmas under mutual influence with the $x$-location of particles in plasma $B$ measured by their distance from the boundary at $d$ (i.e. from 0 to $+\infty$ ). In the sequel, the quantities $\varphi_{A}^{0}, \varphi_{B}^{0}$ (independent of $d$ ) refer similarly to the single semi-infinite plasma $A$ and $B$.
} 
The function $\varphi_{A B}\left(x, x^{\prime}, \mathbf{k}\right)$ (41) describes the correlation between the two plasmas. In terms of the scaled dimensionless variable $\mathbf{q}=\mathbf{k} d$, it has the simple factorized asymptotic behaviour

$$
\begin{aligned}
\varphi_{A B}\left(x, x^{\prime}, \frac{\mathbf{q}}{d}\right) & \sim \frac{1}{d} \frac{4 \pi q}{\kappa_{A} \kappa_{B} \sinh q} \mathrm{e}^{-\kappa_{A}|x|} \mathrm{e}^{-\kappa_{B}\left|x^{\prime}\right|} \\
& =\frac{1}{d} \frac{q}{4 \pi \sinh q} \varphi_{A}^{0}(x, 0, \mathbf{0}) \varphi_{B}^{0}\left(0, x^{\prime}, \mathbf{0}\right), \quad d \rightarrow \infty
\end{aligned}
$$

Finally $\varphi\left(x, x^{\prime}, \mathbf{k}\right)$ obeys the following bound uniformly with respect to $\mathbf{k}$ and $d$ (Appendix $\mathbf{B}$ )

$$
0 \leqslant \varphi\left(x, x^{\prime}, \mathbf{k}\right) \leqslant \varphi^{>}\left(x, x^{\prime}\right) \leqslant \frac{4 \pi}{\kappa}, \quad \kappa d \geqslant 1
$$

The function $\varphi^{>}\left(x, x^{\prime}\right)$ is defined as in (39) with $\varphi_{A A}, \varphi_{A B}$ and $\varphi_{B B}$ replaced by

$$
\begin{gathered}
\varphi_{A A}^{>}\left(x, x^{\prime}\right) \doteqdot \varphi_{B B}^{>}\left(x, x^{\prime}\right) \doteqdot \frac{2 \pi}{\kappa}\left(\mathrm{e}^{-\kappa\left|x-x^{\prime}\right|}+\mathrm{e}^{-\kappa\left|x+x^{\prime}\right|}\right) \\
\varphi_{A B}^{>}\left(x, x^{\prime}\right) \doteqdot \varphi_{B A}^{>}\left(x, x^{\prime}\right) \doteqdot \frac{4 \pi}{\kappa^{2} d} \mathrm{e}^{-\kappa|x|} \mathrm{e}^{-\kappa\left|x^{\prime}\right|}, \quad \kappa:=\min \left\{\kappa_{A}, \kappa_{B}\right\}
\end{gathered}
$$

The Debye-Hückel potential $\Phi\left(x, x^{\prime}, \mathbf{k}\right)$ can be obtained by iterating the integral equation (38). Convergence can be established in the weakcoupling regime:

Lemma (see proof in Appendix B).

Let

$$
r:=\frac{1}{\kappa} \int_{-\infty}^{\infty} d x|u(x)|=\frac{1}{\kappa} \int_{-\infty}^{\infty} d x\left|\kappa^{2}(x)-\bar{\kappa}^{2}(x)\right|
$$

Then for $r<1$ (38) has a solution with the bound

$$
\left|\Phi\left(x, x^{\prime}, \mathbf{k}\right)\right| \leqslant \frac{1}{1-r} \varphi^{>}\left(x, x^{\prime}\right) .
$$

As in (39) we distinguish various contributions according to the location of the arguments $x, x^{\prime}$ of $\Phi\left(x, x^{\prime}, \mathbf{k}\right)$ by setting (see footnote 6 )

$$
\Phi\left(x, x^{\prime}, \mathbf{k}\right)= \begin{cases}\Phi_{A A}\left(x, x^{\prime}, \mathbf{k}\right), & x, x^{\prime}<0, \\ \Phi_{A B}\left(x, x^{\prime}-d, \mathbf{k}\right), & x<0<d<x^{\prime}, \\ \Phi_{B B}\left(x-d, x^{\prime}-d, \mathbf{k}\right), & d<x, x^{\prime},\end{cases}
$$




$$
\rho(\gamma x)= \begin{cases}\rho_{A}(\gamma x), & x<0 \\ \rho_{B}(\gamma, x-d), & x>d .\end{cases}
$$

The quantities $\kappa_{A, B}(x), u_{A, B}(x)$ are defined in the same way. Then, the integral equation (38) splits into

$$
\begin{aligned}
\Phi_{A A}\left(x, x^{\prime}, \mathbf{k}\right)= & \varphi_{A A}\left(x, x^{\prime}, \mathbf{k}\right)-\frac{1}{4 \pi} \int_{-\infty}^{0} d s u_{A}(s) \varphi_{A A}(x, s, \mathbf{k}) \Phi_{A A}\left(s, x^{\prime}, \mathbf{k}\right) \\
& -\frac{1}{4 \pi} \int_{0}^{\infty} d s u_{B}(s) \varphi_{A B}(x, s, \mathbf{k}) \Phi_{B A}\left(s, x^{\prime}, \mathbf{k}\right) . \\
\Phi_{A B}\left(x, x^{\prime}, \mathbf{k}\right)= & \varphi_{A B}\left(x, x^{\prime}, \mathbf{k}\right)-\frac{1}{4 \pi} \int_{-\infty}^{0} d s u_{A}(s) \varphi_{A A}(x, s, \mathbf{k}) \Phi_{A B}\left(s, x^{\prime}, \mathbf{k}\right) \\
& -\frac{1}{4 \pi} \int_{0}^{\infty} d s u_{B}(s) \varphi_{A B}(x, s, \mathbf{k}) \Phi_{B B}\left(s, x^{\prime}, \mathbf{k}\right) .
\end{aligned}
$$

The density profiles depend on $d$ because of the mutual Coulomb interactions between the two plasmas. We shall examine the asymptotic behaviour of $\Phi\left(x, x^{\prime}, \mathbf{k}\right)$ as $d \rightarrow \infty$ under the assumption that these density profiles are uniformly bounded with respect to $d$ and tend to those of single semi-infinite plasmas i.e.

$$
\lim _{d \rightarrow \infty} \rho_{A}(\gamma x)=\rho_{A}^{0}(\gamma x), x<0, \quad \lim _{d \rightarrow \infty} \rho_{B}(\gamma x)=\rho_{B}^{0}(\gamma x), x>0
$$

We denote by $\kappa_{A, B}^{0}(x), u_{A, B}^{0}(x)$ the analogous quantities for the single semi-infinite plasmas. Then one concludes from (52) that uniformly in $\mathbf{k}$

$$
\lim _{d \rightarrow \infty} \Phi_{A A}\left(x, x^{\prime}, \mathbf{k}\right)=\Phi_{A}^{0}\left(x, x^{\prime}, \mathbf{k}\right), \quad x, x^{\prime}<0
$$

where $\Phi_{A}^{0}\left(x, x^{\prime}, \mathbf{k}\right)$ is the Debye-Hückel potential of a semi-infinite plasma in the region $x<0$ determined in terms of the corresponding flat profile potential $\varphi_{A}^{0}\left(x, x^{\prime}, \mathbf{k}\right)(43)$ by

$$
\begin{aligned}
\Phi_{A}^{0}\left(x, x^{\prime}, \mathbf{k}\right)= & \varphi_{A}^{0}\left(x, x^{\prime}, \mathbf{k}\right) \\
& -\frac{1}{4 \pi} \int_{-\infty}^{0} d s u_{A}^{0}(s) \varphi_{A}^{0}(x, s, \mathbf{k}) \Phi_{A}^{0}\left(s, x^{\prime}, \mathbf{k}\right), \quad x, x^{\prime}<0
\end{aligned}
$$

Indeed, in view of the limits (43), (54) and using dominated convergence with the bounds (45), (49) the integral equation (52) reduces to (56) in the limit $d \rightarrow \infty$. One has likewise 


$$
\lim _{d \rightarrow \infty} \Phi_{B B}\left(x, x^{\prime}, \mathbf{k}\right)=\Phi_{B}^{0}\left(x, x^{\prime}, \mathbf{k}\right), \quad x, x^{\prime}>0
$$

where $\Phi_{B}^{0}\left(x, x^{\prime}, \mathbf{k}\right)$ is the Debye-Hückel potential of a semi-infinite plasma in the region $x>0$.

We come now to the correlation $\Phi_{A B}\left(x, x^{\prime}, \frac{\mathbf{q}}{d}\right)$ which is expected to decay as $d^{-1}$ at large separation of the two plasmas. To see this it is useful to write (53) in an alternative form such that $\varphi_{A B}$ appears explicitly in each term of the equation:

$$
\begin{aligned}
\Phi_{A B}\left(x, x^{\prime}, \mathbf{k}\right)= & \varphi_{A B}\left(x, x^{\prime}, \mathbf{k}\right)-\frac{1}{4 \pi} \int_{-\infty}^{0} d s u_{A}(s) \widetilde{\Phi}_{A A}(x, s, \mathbf{k}) \varphi_{A B}\left(s, x^{\prime}, \mathbf{k}\right) \\
& -\frac{1}{4 \pi} \int_{0}^{\infty} d s u_{B}(s) \varphi_{A B}(x, s, \mathbf{k}) \Phi_{B B}\left(s, x^{\prime}, \mathbf{k}\right) \\
& +\left(\frac{1}{4 \pi}\right)^{2} \int_{-\infty}^{0} d s_{1} \int_{0}^{\infty} d s_{2} u_{A}\left(s_{1}\right) u_{B}\left(s_{2}\right) \widetilde{\Phi}_{A A}\left(x, s_{1}, \mathbf{k}\right) \\
& \times \varphi_{A B}\left(s_{1}, s_{2}, \mathbf{k}\right) \Phi_{B B}\left(s_{2}, x^{\prime}, \mathbf{k}\right) .
\end{aligned}
$$

Here $\widetilde{\Phi}_{A A}\left(x, x^{\prime}, \mathbf{k}\right)$ verifies the equation

$$
\widetilde{\Phi}_{A A}\left(x, x^{\prime}, \mathbf{k}\right)=\varphi_{A A}\left(x, x^{\prime}, \mathbf{k}\right)-\frac{1}{4 \pi} \int_{-\infty}^{0} d s u_{A}(s) \varphi_{A A}(x, s, \mathbf{k}) \widetilde{\Phi}_{A A}\left(s, x^{\prime}, \mathbf{k}\right),
$$

which is the equation (52) with the last term omitted. Equation (58) is obtained by iterating (53) and resumming the $\varphi_{A A}$ chains to $\widetilde{\Phi}_{A A}$, or by verifying that it satisfies the basic differential equation (31). By the same arguments that led to (55), it is clear that $\widetilde{\Phi}_{A A}$ also tends to the potential $\Phi_{A}^{0}$ of the semi-infinite plasma.

Introducing the limits (44), (54), (55), (57) in (58) and using again dominated convergence provided by the bounds (47), (46), (49) we find that

$$
\begin{aligned}
\lim _{d \rightarrow \infty} d \Phi_{A B}\left(x, x^{\prime}, \frac{\mathbf{q}}{d}\right)= & \frac{q}{4 \pi \sinh q} \\
& \times\left[\varphi_{A}^{0}(x, 0, \mathbf{0})-\frac{1}{4 \pi} \int_{-\infty}^{0} d s u_{A}^{0}(s) \Phi_{A}^{0}(x, s, \mathbf{0}) \varphi_{A}^{0}(s, 0, \mathbf{0})\right] \\
& \times\left[\varphi_{B}^{0}\left(0, x^{\prime}, \mathbf{0}\right)-\frac{1}{4 \pi} \int_{0}^{\infty} d s u_{B}^{0}(s) \varphi_{B}^{0}(0, s, \mathbf{0}) \Phi_{B}^{0}\left(s, x^{\prime}, \mathbf{0}\right)\right] \\
= & \frac{q}{4 \pi \sinh q} \Phi_{A}^{0}(x, 0, \mathbf{0}) \Phi_{B}^{0}\left(0, x^{\prime}, \mathbf{0}\right)
\end{aligned}
$$


As in (44), the limit factorizes into the product of Debye-Hückel potentials for single semi-infinite plasmas evaluated with one point on the boundary. The last line of (60) follows from (56), the corresponding equation for $\Phi_{B}^{0}\left(x, x^{\prime}, \mathbf{0}\right)$, and the fact that these functions are symmetric in $x, x^{\prime}$.

With this result we can determine the leading term in the asymptotic behaviour of the force (16) in the Debye-Hückel regime. From (29) and (30), one has

$$
\begin{aligned}
\langle f\rangle^{\mathrm{DH}}(d) & =\frac{1}{\beta} \int_{-\infty}^{0} d x \int_{d}^{\infty} d x^{\prime} \int_{0}^{\infty} d k k \mathrm{e}^{-k\left|x-x^{\prime}\right|} \frac{\kappa^{2}(x)}{4 \pi} \frac{\kappa^{2}\left(x^{\prime}\right)}{4 \pi} \Phi\left(x, x^{\prime}, \mathbf{k}\right) \\
& =\frac{1}{\beta d^{2}} \int_{-\infty}^{0} d x \int_{0}^{\infty} d x^{\prime} \int_{0}^{\infty} d q q \mathrm{e}^{-\frac{q}{d}\left|x-x^{\prime}+d\right|} \frac{\kappa_{A}^{2}(x)}{4 \pi} \frac{\kappa_{B}^{2}\left(x^{\prime}\right)}{4 \pi} \Phi_{A B}\left(x, x^{\prime}, \frac{\mathbf{q}}{d}\right)
\end{aligned}
$$

To obtain the second line we have set $\mathbf{k} d=\mathbf{q}$, shifted the $x^{\prime}$-integration by $-d$, and introduced the notation (50), (51). As $d \rightarrow \infty$, one can use (60) and the bounds (46), (49) to conclude again by dominated convergence that

$$
\begin{aligned}
\lim _{d \rightarrow \infty} d^{3}\langle f\rangle^{\mathrm{DH}}(d)= & \frac{1}{8 \pi \beta} \int_{0}^{\infty} d q \frac{4 q^{2} \mathrm{e}^{-q}}{\mathrm{e}^{q}-\mathrm{e}^{-q}}\left(\int_{-\infty}^{0} d x \frac{\left(\kappa_{A}^{0}\right)^{2}(x)}{4 \pi} \Phi_{A}^{0}(x, 0, \mathbf{0})\right) \\
& \times\left(\int_{0}^{\infty} d x^{\prime} \frac{\left(\kappa_{B}^{0}\right)^{2}\left(x^{\prime}\right)}{4 \pi} \Phi_{B}^{0}\left(0, x^{\prime}, \mathbf{0}\right)\right)=\frac{\zeta(3)}{8 \pi \beta}
\end{aligned}
$$

Indeed, because of the charge sum rules (34) for the semi-infinite plasmas, both parentheses are equal to 1 , whereas the $q$ integral yields the value $\zeta(3)$ where $\zeta$ is the Riemann $\zeta$-function.

\section{CONTRIBUTIONS OF THE OTHER GRAPHS}

In this section we show that the single Debye-Hückel bond $F$ saturates the asymptotic behaviour of the force i.e. taking into account the full set of other diagrams does not modify the result (62). For this we use the method of "dressing of the root points" that has been introduced in Ref. 21 to analyse the decay of the quantum truncated charge correlation function. Having singled out the contribution of the single resummed bond $F$, all remaining diagrams constituting $h\left(\gamma \mathbf{r}, \gamma^{\prime} \mathbf{r}^{\prime}\right)$ can be classified into four types, depending on whether their root points are linked to the 
rest of the diagram by a single bond $F$ or not. ${ }^{7}$ We thus write their sum in the form

$$
h^{\mathrm{R}}:=h-F=h^{\mathrm{cc}}+h^{\mathrm{cn}}+h^{\mathrm{nc}}+h^{\mathrm{nn}},
$$

where $h^{\text {cc }}$ stands for the contribution of all graphs that do begin and do end with an $F$ bond (with anything in between), $h^{\mathrm{cn}}$ for the contribution of those that do begin but do not end with an $F$ link, and so on. The latter quantities are obviously related to $h^{\mathrm{nn}}$ by the following integral equations (notations are as in Section 3)

$$
\begin{aligned}
h^{\mathrm{cn}}(a, b) & :=\int d 1 F(a, 1) \rho(1) h^{\mathrm{nn}}(1, b) \\
h^{\mathrm{cc}}(a, b) & :=\int d 1 \int d 2 F(a, 1) \rho(1) h^{\mathrm{nn}}(1,2) \rho(2) F(2, b)
\end{aligned}
$$

and analogously for $h^{\text {nc }}$. Using these representations in (63) together with (20) and building the charge-charge correlation corresponding to $h^{\mathrm{R}}$ according to (27) yields

$$
\begin{aligned}
S^{\mathrm{R}}\left(x, x^{\prime}, \mathbf{k}\right)= & \sum_{\gamma_{1}} \int d x_{1} \sum_{\gamma_{2}} \int d x_{2}\left(\delta\left(x-x_{1}\right)-\frac{\kappa^{2}(x)}{4 \pi} \Phi\left(x, x_{1}, \mathbf{k}\right)\right) \\
& \times e_{\gamma_{1}} \rho\left(\gamma_{1} x_{1}\right) h^{\mathrm{nn}}\left(\gamma_{1} x_{1}, \gamma_{2} x_{2}, \mathbf{k}\right) e_{\gamma_{2}} \rho\left(\gamma_{2} x_{2}\right) \\
& \times\left(\delta\left(x_{2}-x^{\prime}\right)-\frac{\kappa^{2}\left(x^{\prime}\right)}{4 \pi} \Phi\left(x_{2}, x^{\prime}, \mathbf{k}\right)\right) .
\end{aligned}
$$

The function $h^{\mathrm{nn}}\left(\gamma_{1} x_{1}, \gamma_{2} x_{2}, \mathbf{k}\right)$ embodies a resummed contribution, not explicitly known at this point, of higher-order graphs. The only assumption needed on this function in the sequel is integrable clustering uniformly with respect to $d$

$$
\begin{aligned}
\int_{-\infty}^{\infty} d x_{1}\left|h^{\mathrm{nn}}\left(\gamma_{1} x_{1}, \gamma_{2} x_{2}, \frac{\mathbf{q}}{d}\right)\right| & <\infty \\
\int_{-\infty}^{0} d x_{1} \int_{d}^{\infty} d x_{2}\left|h^{\mathrm{nn}}\left(\gamma_{1} x_{1}, \gamma_{2} x_{2}, \frac{\mathbf{q}}{d}\right)\right| & <\infty
\end{aligned}
$$

\footnotetext{
${ }^{7} \mathrm{~A}$ point in a prototype diagram which is linked to the rest of the diagram by exactly one $F$ bond is called a Coulomb point in Ref. 21 .
} 
As a consequence of the bounds (45), (49) the condition (67) obviously holds for the Debye potential $\Phi$, and it is expected to hold for the Ursell function on the ground that as $x_{1} \rightarrow-\infty\left(x_{2} \rightarrow \infty\right) h^{\mathrm{nn}}\left(\gamma_{1} x_{1}, \gamma_{2} x_{2}, \mathbf{k}\right)$ has a fast decay in the bulk part of plasma $A$ (plasma $B$ ). Note that integrating (65) on $x$ (or $x^{\prime}$ ) at $\mathbf{k}=0$ proves the validity of the charge sum rule for the exact charge-correlation function (see (35))

$$
\int_{-\infty}^{\infty} d x \int d \mathbf{y} S\left(x, x^{\prime}, \mathbf{y}\right)=0, \quad S\left(x, x^{\prime}, \mathbf{y}\right)=S^{D H}\left(x, x^{\prime}, \mathbf{y}\right)+S^{R}\left(x, x^{\prime}, \mathbf{y}\right)
$$

Proceeding as in (61) the contribution of $S^{R}\left(x, x^{\prime}, \mathbf{y}\right)$ to the average force can be written in the form (permuting the $x, x^{\prime}$ and $x_{1}, x_{2}$ integrals)

$$
\begin{aligned}
\langle f\rangle^{\mathrm{R}}(d)= & -\frac{1}{d^{2}} \int_{0}^{\infty} d q q \int d x_{1} \int d x_{2} H_{1}\left(x_{1}, \frac{\mathbf{q}}{d}\right) \\
& \times \sum_{\gamma_{1}, \gamma_{2}} e_{\gamma_{1}} e_{\gamma_{2}} \rho\left(\gamma_{1} x_{1}\right) \rho\left(\gamma_{2} x_{2}\right) h^{\mathrm{nn}}\left(\gamma_{1} x_{1}, \gamma_{2} x_{2}, \frac{\mathbf{q}}{d}, d\right) H_{2}\left(x_{2}, \frac{\mathbf{q}}{d}\right)
\end{aligned}
$$

where

$$
\begin{aligned}
& H_{1}\left(x_{1}, \frac{\mathbf{q}}{d}\right)=\int_{-\infty}^{0} d x\left(\delta\left(x-x_{1}\right)-\frac{\kappa^{2}(x)}{4 \pi} \Phi\left(x, x_{1}, \frac{\mathbf{q}}{d}\right)\right) \mathrm{e}^{\frac{\mathbf{q}}{d} x} \\
& H_{2}\left(x_{2}, \frac{\mathbf{q}}{d}\right)=\int_{d}^{\infty} d x^{\prime}\left(\delta\left(x_{2}-x^{\prime}\right)-\frac{\kappa^{2}\left(x^{\prime}\right)}{4 \pi} \Phi\left(x_{2}, x^{\prime}, \frac{\mathbf{q}}{d}\right)\right) \mathrm{e}^{-\frac{\mathbf{q}}{d} x^{\prime}}
\end{aligned}
$$

The behaviour of $\langle f\rangle^{\mathrm{R}}(d)$ as $d \rightarrow \infty$ is determined by that of the functions $H_{1}$ and $H_{2}$, because $h^{\mathrm{nn}}\left(\gamma_{1} x_{1}, \gamma_{2} x_{2}, \frac{\mathbf{q}}{d}, d\right)$ does not vanish in the limit when the variables $x_{1}$ and $x_{2}$ are both located in the same plasma, but tends to the corresponding functions associated with a single semi-infinite plasma. Both $H_{1}$ and $H_{2}$ are $\mathcal{O}(1 / d)$ so that $\langle f\rangle^{\mathrm{R}}(d)=\mathcal{O}\left(1 / d^{4}\right)$ does not contribute to the asymptotic behaviour of the force. More precisely, integrating (31) on $x$ gives for $x_{1}<0$

$$
\begin{aligned}
& \int_{-\infty}^{0} d x\left(\delta\left(x-x_{1}\right)-\frac{\kappa^{2}(x)}{4 \pi} \Phi\left(x, x_{1}, \mathbf{k}\right)\right) \\
& =\int_{d}^{\infty} d x \frac{\kappa^{2}(x)}{4 \pi} \Phi\left(x, x_{1}, \mathbf{k}\right)+\frac{k^{2}}{4 \pi} \int d x \Phi\left(x, x_{1}, \mathbf{k}\right) \\
& =\int_{0}^{\infty} d x \frac{\kappa_{B}^{2}(x)}{4 \pi} \Phi_{B A}\left(x, x_{1}, \mathbf{k}\right)+\frac{k^{2}}{4 \pi} \int d x \Phi\left(x, x_{1}, \mathbf{k}\right)
\end{aligned}
$$


implying with (46), (49)

$$
H_{1}\left(x_{1}, \frac{\mathbf{q}}{d}\right)=\mathcal{O}\left(\frac{1}{d}\right)+\mathcal{O}\left(\frac{q^{2}}{d^{2}}\right), \quad x_{1}<0
$$

For $x_{1}>d$ one has

$$
H_{1}\left(x_{1}, \frac{\mathbf{q}}{d}\right)=-\int_{-\infty}^{0} d x \mathrm{e}^{q x / d} \frac{\kappa^{2}(x)}{4 \pi} \Phi\left(x, x_{1}, \frac{\mathbf{q}}{d}\right)=\mathcal{O}\left(\frac{1}{d}\right) \mathrm{e}^{-\kappa_{B}\left(x_{1}-d\right)}
$$

In the same way

$$
\begin{aligned}
& H_{2}\left(x_{2}, \frac{\mathbf{q}}{d}\right)=\mathcal{O}\left(\frac{\mathrm{e}^{-q}}{d}\right) \mathrm{e}^{-\kappa_{A}\left|x_{2}\right|}, \quad x_{2}<0, \\
& H_{2}\left(x_{2}, \frac{\mathbf{q}}{d}\right)=\mathcal{O}\left(\frac{\mathrm{e}^{-q}}{d}\right)+\mathcal{O}\left(\frac{q^{2} \mathrm{e}^{-q}}{d^{2}}\right), \quad x_{2}>d
\end{aligned}
$$

(the factor $\mathrm{e}^{-q}$ comes from $\mathrm{e}^{-q x / d} \leqslant \mathrm{e}^{-q}$ for $x \geqslant d$ in (70)). Inserting these estimates in the four integration domains determined in (69) by $x_{1}, x_{2}<$ $0, x_{1}, x_{2}>d$ together with the integrability assumptions (66), (67) on $h^{\mathrm{nn}}$ leads to the result

$$
\lim _{d \rightarrow \infty} d^{3}\langle f\rangle(d)=\lim _{d \rightarrow \infty} d^{3}\langle f\rangle^{\mathrm{DH}}(d)+\lim _{d \rightarrow \infty} d^{3}\langle f\rangle^{\mathrm{R}}(d)=\frac{\zeta(3)}{8 \pi \beta}
$$

To conclude this section we present an alternative derivation of the result (75) by selecting the class of diagrams that give the dominant contribution to the Ursell function as $d \rightarrow \infty$. For this we decompose the bond $F\left(\gamma x, \gamma^{\prime} x^{\prime}, \mathbf{k}\right)$ (in Fourier representation) into the sum of four terms according to the location of the arguments $x, x^{\prime}$

$$
\begin{aligned}
F & =F_{A A}+F_{A B}+F_{B A}+F_{B B} \\
F_{A A}\left(\gamma x, \gamma^{\prime} x^{\prime}, \mathbf{k}\right) & = \begin{cases}F\left(\gamma x, \gamma^{\prime} x^{\prime}, \mathbf{k}\right), & x, x^{\prime}<0 \\
0, & \text { otherwise }\end{cases} \\
F_{A B}\left(\gamma x, \gamma^{\prime} x^{\prime}, \mathbf{k}\right) & = \begin{cases}F\left(\gamma x, \gamma^{\prime}, x^{\prime}+d, \mathbf{k}\right), & x<0, x^{\prime}>0 \\
0, & \text { otherwise }\end{cases}
\end{aligned}
$$

with $F_{B A}$ and $F_{B B}$ defined likewise and the similar decomposition for $F^{R}$ ( $x$-integrals in plasma $B$ from now on run in the interval $[0, \infty)$, see footnote 6). The set of prototype graphs is then expanded in a larger set of 
graphs defined in terms of these bonds. It follows from (55) that $F_{A A}$ and $F_{A A}^{R}$ bonds have limits $F_{A}^{0}$ and $F_{A}^{0 R}$ as $d \rightarrow \infty$ where $F_{A}^{0}$ and $F_{A}^{0 R}$ are the bonds corresponding to the semi-infinite plasma $\mathrm{A}$ alone and likewise for $B B$ bonds.

It is shown in Appendix $C$ that the dominant part of the Ursell function $h_{A B}\left(\gamma_{a} \mathbf{r}_{a}, \gamma_{b} \mathbf{r}_{b}\right)$ as $d \rightarrow \infty$ is constituted by the set of graphs that have exactly one $F_{A B}$ bond. This class is obtained by linking the extremity $\gamma_{1} \mathbf{r}_{1}$ of $F_{A B}\left(\gamma_{1} \mathbf{r}_{1}, \gamma_{2} \mathbf{r}_{2}\right)$ to the root point $\gamma_{a} \mathbf{r}_{a}$ of $h_{A B}\left(\gamma_{a} \mathbf{r}_{a}, \gamma_{b} \mathbf{r}_{b}\right)$ in plasma $\Lambda_{A}$ by all possible subgraphs comprising only $A A$ bonds (otherwise one would introduce additional $A B$ bonds), taking into account the excluded convolution rule for $F$ bonds. In the same way the other extremity $\gamma_{2} \mathbf{r}_{2}$ of $F_{A B}\left(\gamma_{1} \mathbf{r}_{1}, \gamma_{2} \mathbf{r}_{2}\right)$ is linked to the root point $\gamma_{b} \mathbf{r}_{b}$ in plasma $\Lambda_{B}$ by all possible subgraphs made of $B B$ bonds. One finds in this way

$$
\begin{aligned}
h_{A B}(a, b, \mathbf{k}) \sim & \int d 1 \int d 2\left[\delta(a, 1)+\left(h_{A A}^{n n}(a, 1, \mathbf{k})+h_{A A}^{c n}(a, 1, \mathbf{k})\right) \rho_{A}(1)\right] \\
& \times F_{A B}(1,2, \mathbf{k})\left[\delta(2, b)+\left(h_{B B}^{n n}(2, b, \mathbf{k})+h_{B B}^{c n}(2, b, \mathbf{k})\right) \rho_{B}(2)\right]
\end{aligned}
$$

Here $a=\left(\gamma_{a} x_{a}\right), 1=\left(\alpha_{1} x_{1}\right), 2=\left(\beta_{2} x_{2}\right), b=\left(\gamma_{b}, x_{b}\right)$, and the integration $\int d 1=\sum_{\alpha_{1}} \int_{-\infty}^{0} d x_{1}$ runs on plasma $A$ and $\int d 2=\sum_{\beta_{2}} \int_{0}^{\infty} d x_{2}$ on plasma $B$. We have also used that the convolution of translation invariant functions in the $\mathbf{y}$-direction is the product of their Fourier transforms. The functions $h_{A A}^{c n}$ and $h_{A A}^{n n}$ are defined as in (63) in terms of $A A$ bonds (similarly for $h_{B B}^{c n}$ and $h_{B B}^{n n}$ in terms of $B B$ bonds). One can write (60) in the form

$$
F_{A B}\left(1,2, \frac{\mathbf{q}}{d}\right) \sim-\frac{q}{4 \pi \beta d \sinh q} \frac{F_{A}^{0}\left(1, a_{0}, \mathbf{0}\right)}{e_{\alpha_{0}}} \frac{F_{B}^{0}\left(b_{0}, 2, \mathbf{0}\right)}{e_{\beta_{0}}}, \quad d \rightarrow \infty
$$

where $a_{0}=\left(\alpha_{0} 0\right)$ indexes a charge $e_{\alpha_{0}}$ located at the boundary $x_{a_{0}}=$ 0 of $\Lambda_{A}$ and $b_{0}$ indexes a charge $e_{\beta_{0}}$ at the boundary of $\Lambda_{B}$. Taking also into account that the functions $h_{A A}^{c n}$ and $h_{A A}^{n n}$ approach the corresponding values $\left(h_{A}^{0}\right)^{c n}$ and $\left(h_{A}^{0}\right)^{n n}$ of a single semi-infinite plasma, one finds that the leading term $\sim 1 / d$ of $h_{A B}\left(\gamma x, \gamma^{\prime} x^{\prime}, \frac{\mathbf{q}}{d}\right)$ factorizes as

$$
h_{A B}\left(a, b, \frac{\mathbf{q}}{d}\right) \sim-\frac{q}{4 \pi \beta d \sinh q} \frac{G_{A}^{0}\left(a, a_{0}\right)}{e_{\alpha_{0}}} \frac{G_{B}^{0}\left(b_{0}, b\right)}{e_{\beta_{0}}}, \quad d \rightarrow \infty
$$


with

$$
\begin{aligned}
G_{A}^{0}\left(a, a_{0}\right) & =F_{A}^{0}\left(a, a_{0}\right)+\int d 1\left[\left(h_{A}^{0}\right)^{\mathrm{nn}}(a, 1)+\left(h_{A}^{0}\right)^{\mathrm{cn}}(a, 1)\right] \rho_{A}^{0}(1) F_{A}^{0}\left(1, a_{0}\right) \\
& =\left(F_{A}^{0}+\left(h_{A}^{0}\right)^{\mathrm{nc}}+\left(h_{A}^{0}\right)^{\mathrm{cc}}\right)\left(a, a_{0}\right)
\end{aligned}
$$

In $G_{A}^{0}$ all functions are evaluated for the single semi-infinite plasma $A$ at $\mathbf{k}=0$ and $\mathbf{k}$ has been omitted from the notation. The expression for $G_{B}^{0}$ is built in the same way. By the same calculation that led to (62) one finds now from (29) that

$$
\begin{aligned}
\lim _{d \rightarrow \infty} d^{3}\langle f\rangle(d)= & \frac{\zeta(3)}{8 \pi \beta}\left(\frac{\int d a e_{\alpha_{a}} \rho_{A}^{0}(a) G_{A}^{0}\left(a, a_{0}\right)}{e_{\alpha_{0}}}\right) \\
& \times\left(\frac{\int d b e_{\beta_{b}} \rho_{B}^{0}(b) G_{B}^{0}\left(b_{0}, b\right)}{e_{\beta_{0}}}\right)
\end{aligned}
$$

It remains to see that both parentheses are equal to -1 because of the electroneutrality sum rule in semi-infinite plasmas. Indeed, using (63) and (64), one recognizes from (80) that

$$
G_{A}^{0}\left(a, a_{0}\right)=h_{A}^{0}\left(a, a_{0}\right)-\int d 1\left[F_{A}^{0}(a, 1) \rho_{A}^{0}(1)+\delta(a, 1)\right]\left(h_{A}^{0}\right)^{n n}\left(1, a_{0}\right) .
$$

The contribution to the force of the second term of (82) involves

$$
\begin{aligned}
& \int \operatorname{dae}_{\alpha_{a}} \rho_{a}^{0}(a)\left[F_{A}^{0}(a, 1) \rho_{A}^{0}(1)+\delta(a, 1)\right] \\
& =e_{\alpha_{1}} \rho\left(\alpha_{1} x_{1}\right)\left(1-\frac{1}{4 \pi} \int_{-\infty}^{0} d x_{a}\left(\kappa_{A}^{0}\right)^{2}\left(x_{a}\right) \Phi_{A}^{0}\left(x_{a}, x_{1}, \mathbf{k}=\mathbf{0}\right)\right)=0
\end{aligned}
$$

which vanishes because of the sum rule (34) in the case of a semi-infinite plasma. The contribution of the first term of (82) is

$$
\int d a e_{\alpha_{a}} \rho_{A}^{0}(a) h_{A}^{0}\left(a, a_{0}\right)=\sum_{\alpha_{a}} \int_{-\infty}^{0} d x_{a} e_{\alpha_{a}} \rho_{A}^{0}\left(\gamma_{a} x_{a}\right) h_{A}^{0}\left(\alpha_{a} x_{a}, \alpha_{0} 0, \mathbf{k}=\mathbf{0}\right)=-e_{\alpha_{0}}
$$


The left-hand side is the total charge of the screening cloud induced in the semi-infinite plasma $\Lambda_{A}$ by the boundary charge $e_{\alpha_{0}}$, which equals $-e_{\alpha_{0}}$ because of perfect screening. ${ }^{(10)}$ By the same considerations the second parenthesis in (81) also equals -1 , hence the final result (75).

\section{PLASMA IN FRONT OF A MACROSCOPIC DIELECTRIC MEDIUM}

In this section we investigate the situation where plasma $B$ is replaced by a semi-infinite macroscopic medium of homogeneous dielectric constant $\epsilon$. The electrostatic potential $V\left(\mathbf{r}, \mathbf{r}^{\prime}\right)$ at $\mathbf{r}$ created by a unit charge at $\mathbf{r}^{\prime} \in$ $\Lambda_{A}$ is the Green function of the Poisson equation with the conditions that the normal component of $\mathbf{D}(x)=\epsilon(x) \mathbf{E}(x),(\epsilon(x)=\epsilon, x \geqslant d, \epsilon(x)=1, x<d)$ and the longitudinal component of $\mathbf{E}(x)$ are continuous at the interface ${ }^{(22)}$

$$
V\left(\mathbf{r}, \mathbf{r}^{\prime}\right)=\left\{\begin{array}{lll}
1 /\left|\mathbf{r}-\mathbf{r}^{\prime}\right|+\Delta /\left|\mathbf{r}-\mathbf{r}^{\prime *}\right|, & x<d, & \Delta=(1-\epsilon) /(1+\epsilon) \\
\widetilde{\Delta} /\left|\mathbf{r}-\mathbf{r}^{\prime}\right|, & x>d, & \widetilde{\Delta}=2 /(1+\epsilon)
\end{array}\right.
$$

where $\mathbf{r}^{*}=(2 d-x, \mathbf{y})$ is the point symmetric to $\mathbf{r}$ with respect to the dielectric surface. The case of an ideal grounded conducting plate $(\epsilon=\infty)$ is formally recovered when $\Delta=-1, \widetilde{\Delta}=0$. In linear electrostatics, the total energy associated to a distribution of charges $\hat{c}(\mathbf{r})$ external to the dielectric is

$$
\frac{1}{8 \pi} \int d \mathbf{r E}(\mathbf{r}) \cdot \mathbf{D}(\mathbf{r})=\frac{1}{2} \int d \mathbf{r} \int d \mathbf{r}^{\prime} \hat{c}(\mathbf{r}) V\left(\mathbf{r}, \mathbf{r}^{\prime}\right) \hat{c}\left(\mathbf{r}^{\prime}\right)
$$

For a configuration of charges $\left\{e_{\alpha_{i}}, \mathbf{r}_{i}\right\}$ in $\Lambda_{A}, \hat{c}(\mathbf{r})=\sum_{i} e_{\alpha_{i}} \delta\left(\mathbf{r}-\mathbf{r}_{i}\right)$, one finds with (85) that the total energy can be written as

$$
\begin{aligned}
U & =U_{A}+U_{A B}, \\
U_{A} & =\sum_{\{i, j\}} \frac{e_{\alpha_{i}} e_{\alpha_{j}}}{\left|\mathbf{r}_{i}-\mathbf{r}_{j}\right|}+v_{\mathrm{SR}}\left(\alpha_{i}, \alpha_{j},\left|\mathbf{r}_{i}-\mathbf{r}_{j}\right|\right), \\
U_{A B} & =\frac{\Delta}{2} \sum_{i=1}^{N} \sum_{j=1}^{N} \frac{e_{\alpha_{i}} e_{\alpha_{j}}}{\mid \mathbf{r}_{i}-\mathbf{r}_{j}^{*}} \mid
\end{aligned}
$$

where we have omitted the (infinite) self-energies of the particles and added a short-range repulsive potential for thermodynamic stability. As in (6), $U_{A B}$ refers to the additional energy due to the presence of the dielectric at distance $d$. The total force exerted by the dielectric on the particles 
of $A$ is obtained by differentiating $U$ with respect to $d$

$$
F_{B \rightarrow \Lambda_{A}}=\frac{\partial}{\partial d} U_{A B}=\Delta \sum_{i=1}^{N} \sum_{j=1}^{N} e_{\alpha_{i}} e_{\alpha_{j}} \frac{x_{i}-x_{j}^{*}}{\left|\mathbf{r}_{i}-\mathbf{r}_{j}^{*}\right|^{3 / 2}} .
$$

It corresponds to the sum of all pairwise forces between charges in the plasma $A$ and their image-charges $\Delta e_{\alpha_{j}}$. Proceeding as in the derivation leading from (7) to (16), the average force along the $x$ direction per unit surface is given by

$$
\begin{aligned}
\langle f\rangle & =\lim _{L \rightarrow \infty} \frac{\left\langle F_{B \rightarrow \Lambda_{A}}\right\rangle_{L}}{L^{2}}=\Delta \int_{-\infty}^{0} d x \int_{-\infty}^{0} d x^{\prime} \int d \mathbf{y} \frac{x-x^{\prime *}}{\left|\mathbf{r}-\mathbf{r}^{\prime *}\right|^{3 / 2}} S\left(x, x^{\prime}, \mathbf{y}\right) \\
& =\frac{-\Delta}{d^{2}} \int_{-\infty}^{0} d x \int_{-\infty}^{0} d x^{\prime} \int \frac{d \mathbf{q}}{(2 \pi)^{2}} 2 \pi \mathrm{e}^{-\frac{q}{d}\left|x+x^{\prime}\right|} \mathrm{e}^{-2 q} S\left(x, x^{\prime}, \frac{\mathbf{q}}{d}\right)
\end{aligned}
$$

To obtain the second line we have introduced the Fourier transform (14), used $x^{\prime *}=2 d-x^{\prime}$, and set $\mathbf{k}=\mathbf{q} / d . S\left(x, x^{\prime}, \mathbf{y}\right)$ is the truncated charge-charge correlation function of the plasma $A$ defined in terms of the statistical weight $\exp (-\beta U)$ associated to the energy (87). The asymptotic analysis of (89) differs from that of the previous section on two points: here the function $\mathrm{S}$ provides a contribution from coincident points to the integrals and we expect $S\left(x, x^{\prime}, \frac{\mathbf{q}}{d}, d\right)$ to tend towards a non-zero limit when $d \rightarrow \infty$, namely

$$
\lim _{d \rightarrow \infty} S\left(x, x^{\prime}, \frac{\mathbf{q}}{d}\right)=S^{0}\left(x, x^{\prime}, \mathbf{0}\right)
$$

with $S^{0}\left(x, x^{\prime}, \mathbf{0}\right)$ the charge correlation of the semi-infinite plasma in absence of the dielectric. Hence, the leading behaviour of the force as $d \rightarrow \infty$

$$
\langle f\rangle \sim\langle f\rangle^{\text {mon. }}+\langle f\rangle^{\text {dip. }}
$$

comes from the first two terms resulting from the expansion of $\exp \{-q \mid x+$ $\left.x^{\prime} \mid / d\right\} \sim 1-q\left|x+x^{\prime}\right| / d$ in (89). At leading order in $\langle f\rangle^{\text {dip. }}$ one can replace $S\left(x, x^{\prime}, \frac{\mathbf{q}}{d}\right)$ by $S^{0}\left(x, x^{\prime}, \mathbf{0}\right)$ so that

$$
\lim _{d \rightarrow \infty} d^{3}\langle f\rangle^{\text {dip. }}=-\Delta \int_{0}^{\infty} d q q^{2} \mathrm{e}^{-2 q} \int_{-\infty}^{0} d x \int_{-\infty}^{0} d x^{\prime}\left(x+x^{\prime}\right) S^{0}\left(x, x^{\prime}, \mathbf{0}\right)=\frac{-\Delta}{16 \pi \beta} .
$$


The first term of the $x, x^{\prime}$ integrals vanishes because of perfect screening whereas the second one equals $1 /(4 \pi \beta)$ as a consequence of the dipole sum rule in a semi-infinite plasma (Ref. 10, formula (3.9), Section C). ${ }^{8}$ Since $\lim _{d \rightarrow \infty} d^{2}\langle f\rangle^{\text {mon. }}=0$ because of perfect screening, one can replace $S\left(x, x^{\prime}, \frac{\mathbf{q}}{d}\right)$ by $S\left(x, x^{\prime}, \frac{\mathbf{q}}{d}\right)-S^{0}\left(x, x^{\prime}, \mathbf{0}\right)$ in the monopole contribution $\langle f\rangle^{\text {mon. }}$. It is convenient to further add and subtract $S^{0}\left(x, x^{\prime}, \frac{\mathbf{q}}{d}\right)$ and to note that:

$$
\begin{gathered}
\lim _{d \rightarrow \infty} d^{3}\left\{\frac{-\Delta}{d^{2}} \int_{-\infty}^{0} d x \int_{-\infty}^{0} d x^{\prime} \int_{0}^{\infty} d q q \mathrm{e}^{-2 q}\left[S^{0}\left(x, x^{\prime}, \frac{\mathbf{q}}{d}\right)-S^{0}\left(x, x^{\prime}, \mathbf{0}\right)\right]\right\} \\
=-\left.\Delta \int_{0}^{\infty} d q q^{2} \mathrm{e}^{-2 q} \frac{d}{d k}\right|_{k=0}\left(\int_{-\infty}^{0} d x \int_{-\infty}^{0} d x^{\prime} S^{0}\left(x, x^{\prime}, k\right)\right)=\frac{-\Delta}{16 \pi \beta} .
\end{gathered}
$$

This follows from the small $k=|\mathbf{k}|$ expansion of the $x, x^{\prime}$ integral that has a linear term $\frac{k}{4 \pi \beta}$ (Ref. 10, formula (3.24), Section C). Collecting (92) and (94) in (91) we see that the large- $d$ behaviour of $\langle f\rangle$ is determined by

$$
\langle f\rangle=\frac{-\Delta}{8 \pi \beta d^{3}}-\frac{\Delta}{d^{2}} \int_{-\infty}^{0} d x \int_{-\infty}^{0} d x^{\prime} \int_{0}^{\infty} d q q \mathrm{e}^{-2 q}\left[S\left(x, x^{\prime}, \frac{\mathbf{q}}{d}\right)-S^{0}\left(x, x^{\prime}, \frac{\mathbf{q}}{d}\right)\right]+\mathrm{o}\left(d^{-3}\right) .
$$

One can now proceed as in Section 3 with Mayer bonds defined in terms of the Green function (85). The Debye-Hückel equation (31) is supplemented with the boundary condition $\lim _{x \rightarrow d_{-}} \partial \Phi\left(x, x^{\prime}, \mathbf{k}\right) / \partial x=$ $\epsilon \lim _{x \rightarrow d_{+}} \partial \Phi\left(x, x^{\prime}, \mathbf{k}\right) / \partial x$, and $\kappa(x)$ is as before for $x \leqslant 0$ but $\kappa(x)=0$ for $x>0$. The solution for piecewise-flat densities is (Ref. 16, formulae (3.2)$(3.5))^{9}$

$$
\varphi\left(x, x^{\prime}, \mathbf{k}\right)=2 \pi \frac{\mathrm{e}^{-k_{A}\left|x-x^{\prime}\right|}}{k_{A}}+2 \pi \frac{\mathrm{e}^{-k_{A}\left|x+x^{\prime}\right|}}{k_{A}} \frac{\left(k_{A}-k\right) \mathrm{e}^{k d}+\Delta\left(k_{A}+k\right) \mathrm{e}^{-k d}}{\left(k_{A}+k\right) \mathrm{e}^{k d}+\Delta\left(k_{A}-k\right) \mathrm{e}^{-k d}} .
$$

It is convenient to single out the potential $\varphi^{0}\left(x, x^{\prime}, \mathbf{k}\right)(43)$ for the semiinfinite plasma in the absence of the dielectric and to split

$$
\varphi\left(x, x^{\prime}, \mathbf{k}\right)=\varphi^{0}\left(x, x^{\prime}, \mathbf{k}\right)+\varphi_{A B}\left(x, x^{\prime}, \mathbf{k}\right),
$$

\footnotetext{
${ }^{8}$ Here the sign is opposite to that in Ref. 10 because the plasma is located in the $x<0$ half space.

${ }^{9}$ Here the minimal distance $d$ between a charge and the dielectric wall plays the role of the hard-core diameter in Ref. 16. Notice that we defined $\Delta$ with the opposite sign and that our plasma fills the region $x<0$.
} 
where, from (43) and (96),

$$
\varphi_{A B}\left(x, x^{\prime}, \mathbf{k}\right)=\frac{8 \pi k \mathrm{e}^{-k_{A}|x|} \mathrm{e}^{-k_{A}\left|x^{\prime}\right|} \Delta \mathrm{e}^{-k d}}{\left(k_{A}+k\right)\left[\left(k_{A}+k\right) \mathrm{e}^{k d}+\left(k_{A}-k\right) \Delta \mathrm{e}^{-k d}\right]}
$$

One observes that $\varphi_{A B}\left(x, x^{\prime}, \mathbf{k}\right)$ has the factorization property analogous to (44)

$$
\begin{aligned}
\lim _{d \rightarrow \infty} d \varphi_{A B}\left(x, x^{\prime}, \frac{\mathbf{q}}{d}\right) & =z(q) \varphi^{0}(x, 0, \mathbf{0}) \varphi^{0}\left(0, x^{\prime}, \mathbf{0}\right), \\
z(q) & =\frac{q}{2 \pi} \frac{\Delta \mathrm{e}^{-2 q}}{1+\Delta \mathrm{e}^{-2 q}} .
\end{aligned}
$$

The potential $\Phi$ corresponding to the exact non-uniform profile is related to $\varphi$ by the integral equation (38). With a reasoning similar to that leading to (58) and (59), it can also be split in two parts

$$
\Phi\left(x, x^{\prime}, \mathbf{k}\right) \equiv \widetilde{\Phi}\left(x, x^{\prime}, \mathbf{k}\right)+\Phi_{A B}\left(x, x^{\prime}, \mathbf{k}\right)
$$

Here $\widetilde{\Phi}$ verifies equation (59) with $\varphi^{0}$ in place of $\varphi_{A A}$ and tends to the potential $\Phi^{0}$ of the semi-infinite plasma in vacuum. $\Phi_{A B}$ solves equation (58) with $u$ in place of $u_{A}$ and $u_{B}, \widetilde{\Phi}$ in place of $\widetilde{\Phi}_{A A}$, and $\Phi$ in place of $\Phi_{B B}$. Then using (99) and the fact that both $\Phi$ and $\widetilde{\Phi}$ tend to $\Phi^{0}$ leads to the asymptotic factorisation of $\Phi_{A B}$

$$
\lim _{d \rightarrow \infty} d \Phi_{A B}\left(x, x^{\prime}, \frac{\mathbf{q}}{d}\right)=z(q) \Phi^{0}(x, 0, \mathbf{0}) \Phi^{0}\left(0, x^{\prime}, \mathbf{0}\right) .
$$

We are now ready to evaluate the force (95) as $d \rightarrow \infty$ in the DebyeHückel approximation. From (27), (24) and (30) one has

$$
S^{\mathrm{DH}}\left(x, x^{\prime}, \frac{\mathbf{q}}{d}\right)=-\frac{1}{\beta} \frac{\kappa^{2}(x)}{4 \pi} \frac{\kappa^{2}\left(x^{\prime}\right)}{4 \pi} \Phi\left(x, x^{\prime}, \frac{\mathbf{q}}{d}\right)+\delta\left(x-x^{\prime}\right) \frac{\kappa^{2}(x)}{4 \pi \beta}
$$

and the analogous relation for $S^{0 \mathrm{DH}}\left(x, x^{\prime}, \frac{\mathbf{q}}{d}\right)$; some care has to be exercised here since coincident points do contribute when both $x, x^{\prime}$ are in the same integration range. We subtract and add $\widetilde{\Phi}\left(x, x^{\prime}, \frac{\mathbf{q}}{d}\right)$ to $\Phi\left(x, x^{\prime}, \frac{\mathbf{q}}{d}\right)$ in (103). This gives two contributions to the force (95). The first one is

$$
\begin{gathered}
\frac{-\Delta}{8 \pi \beta d^{3}}+\frac{\Delta}{\beta d^{2}} \int_{-\infty}^{0} d x \int_{-\infty}^{0} d x^{\prime} \int_{0}^{\infty} d q q \mathrm{e}^{-2 q} \frac{\kappa^{2}(x)}{4 \pi} \frac{\kappa^{2}\left(x^{\prime}\right)}{4 \pi}\left[\Phi\left(x, x^{\prime}, \frac{\mathbf{q}}{d}\right)-\widetilde{\Phi}\left(x, x^{\prime}, \frac{\mathbf{q}}{d}\right)\right] \\
=\frac{-\Delta}{8 \pi \beta d^{3}}+\frac{\Delta}{\beta d^{2}} \int_{-\infty}^{0} d x \int_{-\infty}^{0} d x^{\prime} \int_{0}^{\infty} d q q \mathrm{e}^{-2 q} \frac{\kappa^{2}(x)}{4 \pi} \frac{\kappa^{2}\left(x^{\prime}\right)}{4 \pi} \Phi_{A B}\left(x, x^{\prime}, \frac{\mathbf{q}}{d}\right)
\end{gathered}
$$


The second one involves the quantity

$$
\begin{aligned}
\frac{1}{4 \pi \beta} & {\left[\kappa^{2}(x)-\left(\kappa^{0}\right)^{2}(x)\right] } \\
+ & \frac{-1}{\beta} \int_{-\infty}^{0} d x^{\prime}\left[\frac{\kappa^{2}(x)}{4 \pi} \frac{\kappa^{2}\left(x^{\prime}\right)}{4 \pi} \widetilde{\Phi}\left(x, x^{\prime}, \frac{\mathbf{q}}{d}\right)-\frac{\left(\kappa^{0}\right)^{2}(x)}{4 \pi} \frac{\left(\kappa^{0}\right)^{2}\left(x^{\prime}\right)}{4 \pi} \Phi^{0}\left(x, x^{\prime}, \frac{\mathbf{q}}{d}\right)\right] \\
& =\frac{q^{2}}{4 \pi \beta d^{2}} \int d x^{\prime}\left[\widetilde{\Phi}\left(x, x^{\prime}, \frac{\mathbf{q}}{d}\right)-\Phi^{0}\left(x, x^{\prime}, \frac{\mathbf{q}}{d}\right)\right]=\mathcal{O}\left(\frac{1}{d^{2}}\right) .
\end{aligned}
$$

This equality follows from the relation (33) for $\widetilde{\Phi}$ (relative to $\kappa$ ) and for $\Phi^{0}$ (relative to $\kappa^{0}$ ) since both potentials satisfy the basic differential equation (31). Thus the contribution of (105) to the force is $\mathcal{O}\left(\frac{1}{d^{4}}\right)$. With the factorisation (102) and using the sum rule (34) for $\Phi^{0}$, one finds from (104) and (100) the final result

$$
\lim _{d \rightarrow \infty} d^{3}\langle f\rangle=\frac{-\Delta}{8 \pi \beta}+\frac{\Delta}{\beta} \int_{0}^{\infty} d q q \mathrm{e}^{-2 q} z(q)=\frac{1}{8 \pi \beta} \sum_{n=1}^{\infty} \frac{(-\Delta)^{n}}{n^{3}}
$$

It can be shown along the lines presented in Section 5 that the non meanfield part of the charge correlation function does not contribute to this asymptotic result. According to (101) one splits the bonds $F=\widetilde{F}+F_{A B}$ and $F^{R}=\widetilde{F}^{R}+F_{A B}^{R}$ with $F_{A B}^{R} \equiv \exp \left(F_{A B}-\beta v_{S R}\right)-1-F_{A B}$. The bonds $\widetilde{F}$ and $\widetilde{F}^{R}$ tend to the bonds $F^{0}$ and $F^{0 R}$ pertaining to the semi-infinite plasma without dielectric, whereas $F_{A B}$ vanishes in the limit. At large separation, $F_{A B}^{R} \sim\left(F_{A B}\right)^{2}$ vanishes more rapidly than $F_{A B}$ (see appendix $\mathrm{A}$ ). As in the analysis leading to (79), the leading behaviour of the Ursell function comes from graphs having bonds $\widetilde{F}, \widetilde{F}^{R}$, a single $F_{A B}$ one, and it takes the factorized form (79). The only difference is that both functions $G^{0}$ refer to the same plasma $A$. Then one establishes the validity of (106) as in (81)-(84).

This result coincides with that of Lifshitz. Indeed, a straightforward generalisation of its asymptotic force as $\frac{d}{\beta \hbar c} \gg 1$ (formula (5.5) in Ref. 6) to the case of two different homogeneous dielectric media of constants $\epsilon_{1}, \epsilon_{2}$ yields ${ }^{10}$

$$
f \sim \frac{1}{8 \pi \beta d^{3}} \sum_{n=1}^{\infty} \frac{\left(\Delta_{1} \Delta_{2}\right)^{n}}{n^{3}}, \quad \Delta_{i}=\frac{1-\epsilon_{i}}{1+\epsilon_{i}}, \quad i=1,2 .
$$

\footnotetext{
${ }^{10}$ One generalises formulae (5.2), (5.3) and finally (5.5) of Ref. 6 starting from (2.4) by keep-
} ing $\epsilon_{1}$ and $\epsilon_{2}$ different. 
This reduces to (106) once one of the slabs is a conductor, i.e $\Delta_{1}=-1$. We have therefore provided a derivation of this formula when the conductor is described as a statistical system of fluctuating charges in thermal equilibrium. It is interesting to note that thermal fluctuations in one of the slabs suffice to generate the correct asymptotic value of the force.

\section{APPENDIX A: SLAB OF FINITE THICKNESS}

The analysis of Sections 2-5 applies to infinitely thick slabs. It is interesting to check that the asymptotic behaviour of the force does not depend on the slab thickness. We consider now that the slab $\Lambda_{A}$ has finite thickness $a<\infty$, while, for simplicity, we keep the slab $\Lambda_{B}$ infinitely thick. The setting of Sections 2 and 3 remains the same with the $x$-integration on $\Lambda_{A}$ limited to the interval $-a \leqslant x \leqslant 0$. We then follow the same route as in Sections 4 and 5 by first considering the equation (36) for the piecewise-flat profile

$$
\begin{aligned}
& \bar{\kappa}(x)=0, x<-a, \quad \bar{\kappa}(x)=\frac{1}{a} \int_{-a}^{0} d x \kappa(x) \equiv \kappa_{A},-a<x<0 \\
& \bar{\kappa}(x)=0,0<x<d, \quad \bar{\kappa}(x)=\kappa_{B}, x>d
\end{aligned}
$$

It is convenient to choose $\bar{\kappa}(x)$ equal to the average of $\kappa(x)$ in $\Lambda_{A}$, since we expect the latter to be close to its mean value at weak-coupling.

Solving equation (36) with $x^{\prime}$ fixed, continuity conditions of $x \mapsto$ $\varphi\left(x, x^{\prime}, \mathbf{k}\right)$ and of $x \mapsto \partial_{x} \varphi\left(x, x^{\prime}, \mathbf{k}\right)$ at $x=-a, x=0, x=d$ as well with $\lim _{x \rightarrow \pm \infty} \varphi\left(x, x^{\prime}, \mathbf{k}\right)=0$ yields

$$
\varphi\left(x, x^{\prime}, \mathbf{k}\right)= \begin{cases}\varphi_{A A}\left(x, x^{\prime}, \mathbf{k}\right), & -a<x, x^{\prime}<0 \\ \varphi_{A B}\left(x, x^{\prime}-d, \mathbf{k}\right), & -a<x<0<d<x^{\prime} \\ \varphi_{B B}\left(x-d, x^{\prime}-d, \mathbf{k}\right), & d<x, x^{\prime}\end{cases}
$$

where

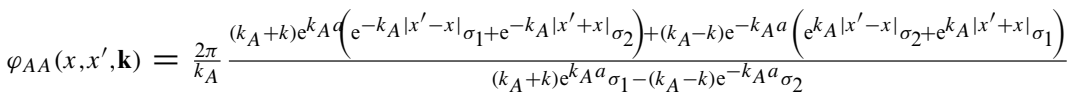

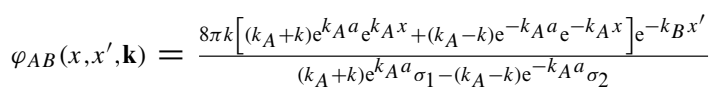

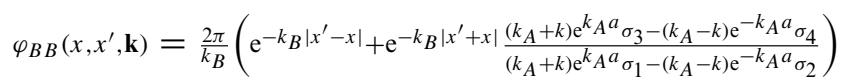


and

$$
\begin{array}{ll}
\sigma_{1}=\left(k_{A}+k\right)\left(k_{B}+k\right) \mathrm{e}^{k d}-\left(k_{A}-k\right)\left(k_{B}-k\right) \mathrm{e}^{-k d} & \sigma_{2}=\left(k_{A}-k\right)\left(k_{B}+k\right) \mathrm{e}^{k d}-\left(k_{A}+k\right)\left(k_{B}-k\right) \mathrm{e}^{-k d} \\
\sigma_{3}=\left(k_{A}+k\right)\left(k_{B}-k\right) \mathrm{e}^{k d}-\left(k_{A}-k\right)\left(k_{B}+k\right) \mathrm{e}^{-k d} & \sigma_{4}=\left(k_{A}-k\right)\left(k_{B}-k\right) \mathrm{e}^{k d}-\left(k_{A}+k\right)\left(k_{B}+k\right) \mathrm{e}^{-k d} \\
k_{A}=\sqrt{k^{2}+\kappa_{A}^{2}} & k_{B}=\sqrt{k^{2}+\kappa_{B}^{2}} .
\end{array}
$$

One deduces also from the differential equations that for any $a>0$ both $\varphi$ and $\Phi$ verify the charge sum rule (34). As $a \rightarrow \infty$, formulae reduce to those obtained in Section 4 for two semi-infinite plasmas.

The main observation to be made on this explicit result is that it obeys exactly the same factorisation property in terms of the scaled variable $\mathbf{q}=\mathbf{k} d$ as (44) with the the same factors (here $\varphi_{A}^{0}(x, 0, \mathbf{0})$ corresponds to the single plasma $A$ with finite thickness). The rest of the analysis is the same as in Section 4, with the difference that the above solution verifies $a$-dependent bounds in place of (45)-(47), namely (Appendix B)

$$
\left|\varphi\left(x, x^{\prime}, \mathbf{k}\right)\right| \leqslant \varphi^{>}\left(x, x^{\prime}\right) \leqslant \frac{4 \pi}{\kappa} \operatorname{coth} \kappa a, \quad \kappa d \geqslant 1,
$$

where $\varphi^{>}\left(x, x^{\prime}\right)$ is defined piecewise from $\varphi_{A A}^{>}, \varphi_{A B}^{>}$, etc. as in (A.2) with

$$
\begin{aligned}
\varphi_{A A}^{>}\left(x, x^{\prime}\right) & =\frac{2 \pi}{\kappa} \frac{\cosh \kappa\left(a-\left|x^{\prime}-x\right|\right)+\cosh \kappa\left(a-\left|x^{\prime}+x\right|\right)}{\sinh \kappa a} \\
\varphi_{A B}^{>}\left(x, x^{\prime}\right) & =\frac{4 \pi}{\kappa^{2} d} \frac{\cosh \kappa(a-|x|)}{\sinh \kappa a} \mathrm{e}^{-\kappa x^{\prime}} \\
\varphi_{B B}^{>}\left(x, x^{\prime}\right) & =\frac{2 \pi}{\kappa}\left(\mathrm{e}^{-\kappa\left|x^{\prime}-x\right|}+\mathrm{e}^{-\kappa\left|x^{\prime}+x\right|}\right), \quad \kappa:=\min \left\{\kappa_{A}, \kappa_{B}\right\}
\end{aligned}
$$

The potential $\Phi$ with structured profiles is related to $\varphi$ by the integral equation (38) and the estimate (49) of the lemma becomes

$$
\left|\Phi\left(x, x^{\prime}, \mathbf{k}\right)\right| \leqslant \frac{1}{1-r(a)} \varphi^{>}\left(x, x^{\prime}\right), \quad r(a):=r \operatorname{coth} \kappa a
$$

where $r$ is defined by (48). To have $r(a)<1$ one needs $r$ sufficiently small (weak-coupling, see Appendix B) and $\kappa a$ not too small, i.e. the slab width is larger than the typical screening length in the plasma. ${ }^{11}$ Then the steps

\footnotetext{
${ }^{11}$ Notice that the above bounds cannot be uniform in $a: \varphi_{A}^{0}\left(x, x^{\prime}, \mathbf{0}\right)$ diverges as $a \rightarrow 0$ so that its integral over $[-a, 0]$ leads to the constant value $\frac{4 \pi}{\kappa_{A}}$ requested by the charge sum rule.
} 
leading to (62) are the same as in Section 4 and the considerations of Section 5 apply as well. The reason for the asymptotic force being independent of the slab thickness is clearly displayed in expressions (62) and (81): it only depends on the screening cloud associated to charges located at the inner boundaries of the slabs, and thus is insensitive to charge fluctuations elsewhere in the slabs.

\section{APPENDIX B: BOUNDS FOR THE DEBYE-HÜCKEL POTENTIAL}

In this appendix, we present some details of the calculations leading to bounds used throughout the paper for the Debye-Hückel potentials $\varphi$ and $\Phi$ and discuss the validity of (49) in the weak-coupling regime.

At first, we show the bound (A.7), which is a generalisation of (45) to the case where plasma $A$ is of finite thickness $a$. Result (45) is recovered by taking $a \rightarrow \infty$. From (A.6), one has $\sigma_{1} \geqslant \sigma_{2} ; \sigma_{1} \geqslant \kappa_{A} \kappa_{B}\left(\mathrm{e}^{k d}-\mathrm{e}^{-k d}\right)$, and $\sigma_{2}+\sigma_{4} \leqslant \sigma_{1}+\sigma_{3} ; \sigma_{3}-\sigma_{1} \leqslant \sigma_{4}-\sigma_{2}<0$. This implies

$$
-1 \leqslant \frac{\left(k_{A}+k\right) \mathrm{e}^{k_{A} a} \sigma_{3}-\left(k_{A}-k\right) \mathrm{e}^{-k_{A} a} \sigma_{4}}{\left(k_{A}+k\right) \mathrm{e}^{k_{A} a} \sigma_{1}-\left(k_{A}-k\right) \mathrm{e}^{-k_{A} a} \sigma_{2}} \leqslant 1,
$$

which yields the bound (A.10) for $\varphi_{B B}$ :

$$
\left|\varphi_{B B}\right| \leqslant \frac{2 \pi}{k_{B}}\left(\mathrm{e}^{-k_{B}\left|x^{\prime}-x\right|}+\mathrm{e}^{-k_{B}\left|x^{\prime}+x\right|}\right) \leqslant \varphi_{B B}^{>}\left(x, x^{\prime}\right) .
$$

To obtain the bound (A.9) for $\varphi_{A B}$, we then note that

$$
\sigma_{1}-\frac{k_{A}-k}{k_{A}+k} \mathrm{e}^{-2 k_{A} a} \sigma_{2} \geqslant\left(1-\mathrm{e}^{-2 k_{A} a}\right) \sigma_{1} .
$$

Thus, $\frac{k}{\sigma_{1}-\frac{k_{A}-k}{k_{A}+k} \mathrm{e}^{-2 k_{A} a} \sigma_{2}} \leqslant \frac{1}{1-\mathrm{e}^{-2 k_{A} a}} \frac{1}{\kappa_{A} \kappa_{B} d} \frac{k d}{\mathrm{e}^{k d}-\mathrm{e}^{-k d}} \leqslant \frac{1}{1-\mathrm{e}^{-2 k_{A} a}} \frac{1}{\kappa^{2} d} \frac{1}{2}$, so that

$$
0 \leqslant \varphi_{A B}\left(x, x^{\prime}, \mathbf{k}\right) \leqslant \frac{4 \pi}{\kappa^{2} d} \frac{\mathrm{e}^{k_{A}(a-|x|)}+\mathrm{e}^{-k_{A}(a-|x|)}}{\mathrm{e}^{k_{A} a}-\mathrm{e}^{-k_{A} a}} \mathrm{e}^{-k_{B} x^{\prime}} \leqslant \varphi_{A B}^{>}\left(x, x^{\prime}\right) .
$$

Last inequality uses

$$
\frac{\mathrm{e}^{k x}+\mathrm{e}^{-k x}}{\mathrm{e}^{k X}-\mathrm{e}^{-k X}} \leqslant \frac{\mathrm{e}^{\kappa x}+\mathrm{e}^{-\kappa x}}{\mathrm{e}^{\kappa X}-\mathrm{e}^{-\kappa X}}, \quad 0<x \leqslant X, \quad k \geqslant \kappa>0 .
$$


Finally, (B.1), the fact that $\left|\sigma_{2} / \sigma_{1}\right| \leqslant 1$ and (B.2) show the bound (A.8)

$$
\begin{aligned}
\left|\varphi_{A A}\left(x, x^{\prime}, \mathbf{k}\right)\right| & \leqslant \frac{2 \pi}{\kappa_{A}} \frac{\sigma_{1} \mathrm{e}^{-k_{A}\left|x^{\prime}-x\right|}+\left|\sigma_{2}\right| \mathrm{e}^{-k_{A}\left|x^{\prime}+x\right|}+\mathrm{e}^{-2 k_{A} a}\left(\left|\sigma_{2}\right| \mathrm{e}^{k_{A}\left|x^{\prime}-x\right|}+\sigma_{1} \mathrm{e}^{k_{A}\left|x^{\prime}+x\right|}\right)}{\left(1-\mathrm{e}^{-2 k_{A} a}\right) \sigma_{1}} \\
& \leqslant \varphi_{A A}^{>}\left(x, x^{\prime}\right) .
\end{aligned}
$$

\section{B.1. Proof of the Lemma}

To proof the bound (49) of $\Phi\left(x, x^{\prime}, \mathbf{k}\right)$, we proceed as follows. By (38) we can develop $\Phi\left(x, x^{\prime}, \mathbf{k}\right)$ as a perturbation series w.r.t. $\varphi\left(x, x^{\prime}, \mathbf{k}\right)$, whose $n^{\text {th }}$ term reads

$$
\left(\frac{-1}{4 \pi}\right)^{n} \int d s_{1} \cdots d s_{n} u\left(s_{1}\right) \cdots u\left(s_{n}\right) \varphi\left(x, s_{1}, \mathbf{k}\right) \varphi\left(s_{1}, s_{2}, \mathbf{k}\right) \cdots \varphi\left(s_{n}, x^{\prime}, \mathbf{k}\right)
$$

This term is bounded by $r^{n} \varphi^{>}\left(x, x^{\prime}\right)$, where $r$ is given by (48). Indeed, according to $(45), \varphi\left(x, x^{\prime}, \mathbf{k}\right)$ is bounded by $\varphi^{>}\left(x, x^{\prime}\right)$, which itself satisfies

$$
\varphi^{>}(x, s) \varphi^{>}\left(s, x^{\prime}\right) \leqslant \frac{4 \pi}{\kappa} \varphi^{>}\left(x, x^{\prime}\right), \quad \forall s, x, x^{\prime}
$$

Consequently, if $r<1$, the series is absolutely convergent and the lemma holds. Inequality (B.4) is proven using (46), (47) and verifying it for each case. As an example,

$$
\begin{aligned}
\varphi_{A A}^{>}(x, s) \varphi_{A B}^{>}\left(s, x^{\prime}\right) & =\frac{2 \pi}{\kappa} \frac{4 \pi}{\kappa^{2} d} \mathrm{e}^{-\kappa\left|x^{\prime}\right|}\left(\mathrm{e}^{-\kappa(|x-s|+|s|)}+\mathrm{e}^{-\kappa(|x+s|+|s|)}\right) \\
& \leqslant \frac{4 \pi}{\kappa} \varphi_{A B}^{>}\left(x, x^{\prime}\right),
\end{aligned}
$$

because $|x \pm s|+|s| \geqslant|x|$. Some of the majorations leading to (B.4) assume $\kappa d \geqslant 1$.

If plasma $A$ is finitely thick, (B.4) generalizes to

$$
\varphi^{>}(x, s) \varphi^{>}\left(s, x^{\prime}\right) \leqslant \frac{4 \pi}{\kappa} \operatorname{coth}(\kappa a) \varphi^{>}\left(x, x^{\prime}\right), \quad \forall x, x^{\prime}, s
$$

and we obtain the bound (A.11) for $\Phi$. 


\section{B.2. Profiles in Weakly Coupled Plasmas}

The parameter $r$ occurring in the bound (49) can be chosen small enough in the weak-coupling regime, defined by $\Gamma=\frac{1}{2} \beta e^{2} \kappa \ll 1(e$ is a typical charge of the system). Indeed, to estimate $r$, the deviations of the density profiles to their bulk values need to be known. In the simplest case of a semi-infinite chargesymmetric plasma $^{12}$ in the weak-coupling regime, Jancovici ${ }^{(20)}$ finds

$$
\frac{\rho_{A}(\gamma x)-\rho_{A \gamma}}{\rho_{A \gamma}} \simeq \frac{\beta e_{\gamma}^{2}}{2} \kappa_{A} \chi\left(\kappa_{A} x\right), \quad \Gamma \ll 1,
$$

where $\chi$ is integrable. Integrating over $x<0$ and forming $r$ according to (48) shows that $r$ is proportional to $\Gamma$. This also holds if the semi-infinite plasma is not charge-symmetric (see Ref. 16, section 5). For our two-plasmas system, $r$ will be less than 1 provided $\Gamma$ is small and $d$ is large.

\section{APPENDIX C: DECAY OF MAYER GRAPHS AT LARGE SLAB SEPARATION}

We consider prototype graphs constituted of bonds (76) labelled by the indices $A A, A B, B A, B B$ according to the respective location of the variables $x, x^{\prime}$ in slab $A$ or in slab $B$. In view of (29) and after the changes $\mathbf{k} d=\mathbf{q}, x^{\prime} \rightarrow x^{\prime}-d$, the contribution to the force of a graph $\Pi_{A B}$ with first root point in $\Lambda_{A}$ and second root point in $\Lambda_{B}$ is

$$
\begin{aligned}
\langle f\rangle^{\Pi_{A B}}= & -\frac{1}{d^{2}} \int_{-\infty}^{0} d x \int_{0}^{\infty} d x^{\prime} \int_{0}^{\infty} d q q \mathrm{e}^{-\frac{q}{d}\left|x-x^{\prime}\right|} \mathrm{e}^{-q} \\
& \times \sum_{\gamma, \gamma^{\prime}} e_{\gamma} e_{\gamma^{\prime}} \rho_{A}(\gamma x) \rho_{B}\left(\gamma^{\prime} x^{\prime}\right) \pi_{A B}\left(\gamma x, \gamma^{\prime} x^{\prime}, \frac{\mathbf{q}}{d}\right) .
\end{aligned}
$$

A graph $\Pi_{A B}$ having $L$ bonds of $\mathcal{F}_{A B}$ or $\mathcal{F}_{B A}$ type, written in Fourier space with respect to the $\mathbf{y}$-variables, is of the general form

$$
\begin{aligned}
\Pi_{A B}\left(\gamma x, \gamma^{\prime} x^{\prime}, \mathbf{k}\right)= & \frac{1}{S_{\pi_{A B}}} \int d 1 \rho(1) \cdots \int d m \rho(m) \int \prod_{j=1}^{L} \frac{d \boldsymbol{\ell}_{j}}{(2 \pi)^{2}} \mathcal{F}_{[A B]}\left(\boldsymbol{\ell}_{j}\right) \\
& \times \int \prod_{a} \frac{d \mathbf{k}_{a}}{(2 \pi)^{2}} \mathcal{F}_{A A}\left(\mathbf{k}_{a}\right) \int \prod_{b} \frac{d \mathbf{k}_{b}}{(2 \pi)^{2}} \mathcal{F}_{B B}\left(\mathbf{k}_{b}\right) \\
& \times \prod_{n=0}^{m}(2 \pi)^{2} \delta\left[\mathbf{k},\left\{\boldsymbol{\ell}_{j}\right\}_{n},\left\{\mathbf{k}_{a}\right\}_{n},\left\{\mathbf{k}_{b}\right\}_{n}\right] .
\end{aligned}
$$

\footnotetext{
${ }^{12} \mathrm{~A}$ charge-symmetric plasma has two species with opposite charges and same bulk densities.
} 
Here $m$ is the number of internal points, $i=\left(\gamma_{i}, x_{i}\right)$ and $\int d i$ stands for summation over particle species and integration over $x_{i} . \mathcal{F}(\mathbf{k})$ stands either for $F(\mathbf{k})$ or $F^{\mathrm{R}}(\mathbf{k})$ and we have omitted the species and $x, x^{\prime}$ dependencies from the notation. ${ }^{13}$ The product of $m+1 \delta$-functions expresses the conservation of wave numbers at the $m$ internal points plus a relation that fixes the sum of ingoing (or outgoing) wave numbers to $\mathbf{k}$, as a result of $\mathbf{y}$-translation invariance. These constitute $m+1$ linear equations between wave numbers from the sets $\left\{\boldsymbol{\ell}_{j}\right\},\left\{\mathbf{k}_{a}\right\},\left\{\mathbf{k}_{b}\right\}$, which imply $C$ independent relations involving only $\boldsymbol{\ell}$ variables. Depending on the topology of the graph, $1 \leqslant C \leqslant L$. Consider, e.g., the graph constituted by a single chain of bonds $F_{A B}\left(\boldsymbol{\ell}_{1}\right) F_{B B}^{R}\left(\mathbf{k}_{b}\right) F_{B A}\left(\boldsymbol{\ell}_{2}\right) F_{A A}^{R}\left(\mathbf{k}_{a}\right) F_{B A}\left(\boldsymbol{\ell}_{3}\right)$ with $L=3 .{ }^{14}$ The conservation laws $\mathbf{k}=\boldsymbol{\ell}_{1}=\mathbf{k}_{a}=\boldsymbol{\ell}_{2}=\mathbf{k}_{b}=\boldsymbol{\ell}_{3}$ imply the independent relations $\mathbf{k}=\boldsymbol{\ell}_{1}, \boldsymbol{\ell}_{1}=\boldsymbol{\ell}_{2}, \boldsymbol{\ell}_{2}=\boldsymbol{\ell}_{3}$ between the $\boldsymbol{\ell}$ variables, thus $C=3$. Consider now the graph constituted of two parallel chains $F_{A A}^{R}\left(\mathbf{k}_{a 1}\right) F_{A B}\left(\ell_{1}\right) F_{B B}^{R}\left(\mathbf{k}_{b 1}\right)$ and $F_{A A}^{R}\left(\mathbf{k}_{a 2}\right) F_{A B}\left(\boldsymbol{\ell}_{2}\right) F_{B B}^{R}\left(\mathbf{k}_{b 2}\right)$ with $L=2$. The conservation laws are $\mathbf{k}=\mathbf{k}_{a 1}+$ $\mathbf{k}_{a 2}, \mathbf{k}_{a 1}=\boldsymbol{\ell}_{1}=\mathbf{k}_{b 1}, \mathbf{k}_{a 2}=\boldsymbol{\ell}_{2}=\mathbf{k}_{b 2}$ implying the single relation $\mathbf{k}=\boldsymbol{\ell}_{1}+\boldsymbol{\ell}_{2}$, thus $C=1$.

Then we perform the integrations in (C.2) in the following order. We first carry $C$ integrals on the $\delta$ functions corresponding to the above relations between $\ell$ variables: this expresses the $\ell_{j}$ variables $j=1, \ldots, L$ in the integrand as linear combinations of the remaining $L-C$ ones, say $\boldsymbol{\ell}_{C+1}, \ldots, \boldsymbol{\ell}_{L}$. We evaluate now $\Pi_{A B}$ at $\mathbf{k}=\mathbf{q} / d$ and change the variables $\boldsymbol{\ell}_{j}=\mathbf{q}_{j} / d, j=C+1, \ldots, L$ : the Jacobian provides a factor $d^{-2(L-C)}$. As $d \rightarrow \infty$ the $\mathbf{k}_{a}, \mathbf{k}_{b}$ and $\mathbf{q}$ integrals factorize. Indeed in $\mathcal{F}_{A A}$ or $\mathcal{F}_{B B}$ bonds the $\mathbf{q}$ dependences occur in the form $\mathbf{q} / d \rightarrow 0, d \rightarrow \infty$ whereas for $\mathcal{F}_{A B}$ or $\mathcal{F}_{B A}$ bonds we use the asymptotic form (60). This yields a factor $d^{-n[A B]}$ if the number of $F_{[A B]}$ bonds is $n[A B]$ and $d^{-4 n^{\mathrm{R}}[A B]}$ if the number of $F_{[A B]}^{\mathrm{R}}$ bonds is $n^{\mathrm{R}}[A B]$ (for the latter, see at the end of this appendix). Then the $\mathbf{k}_{a}$ and $\mathbf{k}_{b}$ integrals refer to products of $\mathcal{F}_{A A}$ and $\mathcal{F}_{B B}$ as in single semiinfinite plasmas and the $\mathbf{q}$ integrals are carried on product of functions $q / \sinh q$. For the above examples the $\mathbf{q}$ integrals are $\int d \mathbf{q}(q / \sinh q)^{3}$ and $\int d \mathbf{q}_{1} \int d \mathbf{q}_{2}\left(q_{1} / \sinh q_{1}\right)\left(\left|\mathbf{q}_{1}-\mathbf{q}_{2}\right| / \sinh \left|\mathbf{q}_{1}-\mathbf{q}_{2}\right|\right)$. As a final result a graph decays as

$$
d^{-2(L-C)} d^{-n[A B]} d^{-4 n^{\mathrm{R}}[A B]}, \quad d \rightarrow \infty
$$

times a factor of order one resulting of the above integrals. It is clear that the minimal decay $d^{-1}$ is obtained when there is only one $F_{A B}$ bond.

\footnotetext{
${ }^{13}$ In (C.2), $\mathcal{F}_{[A B]}$ designates either a $\mathcal{F}_{A B}$ or a $\mathcal{F}_{B A}$ bond. Notice that $\mathcal{F}_{B A}(i, j, \mathbf{k})=$ $\mathcal{F}_{A B}(j, i, \mathbf{k})$.

${ }^{14}$ The $F^{R}$ bonds are needed because of the excluded convolution rule between $F$ bonds.
} 
It remains to examine the decay of a $F_{[A B]}^{\mathrm{R}}$ bond, which reads in Fourier form, according to (21)

$$
F_{A B}^{\mathrm{R}}(\mathbf{k})=\int d \mathbf{y} e^{-i \mathbf{k} \cdot \mathbf{y}}\left[\exp \left(-\beta e_{1} e_{2} \Phi_{A B}(\mathbf{y})\right)-1+\beta e_{1} e_{2} \Phi_{A B}(\mathbf{y})\right]
$$

The $x$ variables are omitted and $\left|x_{1}-x_{2}\right|$ is large enough so that the short range part of the potential does not contribute. In view of $(60), \Phi_{A B}(\mathbf{y})$ has the asymptotic form

$$
\begin{aligned}
\Phi_{A B}(\mathbf{y}) & =\frac{1}{d^{2}} \int \frac{d \mathbf{q}}{(2 \pi)^{2}} \exp \left(i \frac{\mathbf{q}}{d} \cdot \mathbf{y}\right) \Phi_{A B}\left(\frac{\mathbf{q}}{d}\right) \\
& \sim \frac{1}{d^{3}} f\left(\frac{\mathbf{y}}{d}\right) \quad \text { with } \quad f(\mathbf{y})=\Phi_{A}^{0} \Phi_{B}^{0} \int \frac{d \mathbf{q}}{(2 \pi)^{2}} e^{i \mathbf{q} \cdot \mathbf{y}} \frac{q}{4 \pi \sinh q} .
\end{aligned}
$$

Hence substituting (C.5) in (C.4) and expanding for large $d$ after the change of variable $\mathbf{u}=\mathbf{y} / d$ gives

$$
\begin{aligned}
F_{A B}^{\mathrm{R}}\left(\frac{\mathbf{q}}{d}\right) & \sim d^{2} \int d \mathbf{u} e^{-i \mathbf{q} \cdot \mathbf{u}}\left[\exp \left(-\frac{\beta}{d^{3}} f(\mathbf{u})\right)-1+\frac{\beta}{d^{3}} f(\mathbf{u})\right] \\
& \sim \frac{1}{d^{4}} \int d \mathbf{u} e^{-i \mathbf{q} \cdot \mathbf{u}}(f(\mathbf{u}))^{2}=\mathcal{O}\left(\frac{1}{d^{4}}\right) .
\end{aligned}
$$

\section{ACKNOWLEDGEMENTS}

We thank F. Cornu and B. Jancovici for useful discussions.

\section{REFERENCES}

1. P. Milonni, The Quantum Vacuum: An Introduction to Quantum Electrodynamics (Academic Press, San Diego, 1994).

2. V. M. Mostepanenko and N. N. Trunov, The Casimir Effect and its Applications (Clarendon Press, Oxford, 1997).

3. G. Plunien, B. Müller and W. Greiner, The Casimir effect, Phys. Reports 134:87-193 (1986).

4. B. Duplantier and V. Rivasseau, Poincaré Seminar 2002: Vacuum Energy-Renormalization (Progress in Math. Phys., V30.) (Birkhäuser, Basel, 2003).

5. R. Balian and B. Duplantier, Electromagnetic Waves Near Perfect Conductors, I. Multiple Scattering Expansions and Distribution of Modes, Ann. Phys. 104:300-335 (1977); II. Casimir Effect, Ann. Phys. 112:165-208 (1978).

6. E. M. Lifshitz, The theory of molecular attractive forces between solids, J. Exp. Th. Phys. USSR 29:94-110 (1955). 
7. E. M. Lifshitz, L. D. Landau and L. P. Pitaevskii, Electrodynamics of Continuous Media (Landau course, vol. 8, Pergamon Press, Oxford, 1984), §90; I. E. Dzyaloshinskii, E. M. Lifshitz, L. P. Pitaevskii, The General Theory of Van der Waals Forces, Adv. Phys. 10:165-209 (1961).

8. J. Schwinger, Casimir effect in source theory, Lett. Math. Phys. 1:43-47 (1975).

9. J. Schwinger, L. L. DeRaad Jr. and K. A. Milton, Casimir effect in dielectrics, Ann. Phys. 115:1-23 (1978).

10. Ph. A. Martin, Sum rules in charged fluids, Rev. Mod. Phys. 60:1075-1127 (1988).

11. P. J. Forrester, B. Jancovici and G. Téllez, Universality in some classical Coulomb systems of restricted dimension, J. Stat. Phys. 84:359-378 (1996).

12. B. Jancovici and G. Téllez, The ideal conductor limit, J. Phys. A: Math. Gen. 29:11551166 (1996).

13. B. Jancovici and L. Šamaj, Screening of Casimir forces by electrolytes in semi-infinite geometries, University of Paris-Sud preprint, submitted to JSTAT.

14. E. Meeron, Theory of potentials of average force and radial distribution function in ionic solutions, J. Chem. Phys. 28:630-643 (1958); E. Meeron, Plasma Physics (McGrawHill, New York, 1961).

15. J.-N. Aqua and F. Cornu, Dipolar effective interaction in a fluid of charged spheres near a dielectric plate, Phys. Rev. E 68:026133, 1-17 (2003).

16. J.-N. Aqua and F. Cornu, Density profiles in a classical Coulomb fluid near a dielectric wall, I. Mean-field scheme J. Stat. Phys 105:211-244 (2001).

17. J.-N. Aqua and F. Cornu, Density profiles in a classical Coulomb fluid near a dielectric wall, II. Weak-coupling systematic expansion, J. Stat. Phys. 105:245-283 (2001).

18. J.-P. Hansen and I. R. McDonald, Theory of simple liquids (Second edition, Academic Press, London, 1986).

19. R. L. Guernsey, Correlation effects in semi-infinite plasmas, Phys. Fluids 13:2089-2102 (1970).

20. B. Jancovici, Classical coulomb systems near a plane wall. I, J. Stat. Phys. 28:43-65 (1982).

21. F. Cornu, Correlations in Quantum Plasmas. II. Algebraic Tails, Phys. Rev. E 53:45954631 (1996). See also D. C. Brydges, Ph. A. Martin, Coulomb systems at low density: a review, J. Stat. Phys 96:1163-1330 (1999), sect. VI.A.3.

22. J. D. Jackson, Classical Electrodynamics (Wiley Text Books, New York, 1998); J. Schwinger, L. L. DeRaad Jr., K. A. Milton and W.-Y. Tsai, Classical Electrodynamics (Westview Press, Boulder, 1998). 